\title{
Absence of localized edge modes in spite of a non-trivial Zak phase in $\mathrm{BiCu}_{2} \mathrm{PO}_{6}$
}

\author{
M. Malki, ${ }^{*}$ L. Müller, and G. S. Uhrig $\odot$ \\ Lehrstuhl für Theoretische Physik 1, TU Dortmund, 44221 Dortmund, Germany
}

(Received 12 July 2019; published 23 December 2019)

\begin{abstract}
Topological properties of physical systems have attracted tremendous interest in condensed matter and atomic physics in the last decade. Recently, magnetic solid state compounds with and without magnetic order have become a focus. We provide evidence that $\mathrm{BiCu}_{2} \mathrm{PO}_{6}$ is a gapful, disordered quantum antiferromagnet with a non-trivial finite Zak phase, which characterizes one-dimensional systems. Our calculations show that in spite of the non-trivial topology no localized edge modes occur. This unexpected behavior is explained by a generic feature, namely the significance of direct and indirect gaps in topological systems.
\end{abstract}

DOI: 10.1103/PhysRevResearch.1.033197

\section{INTRODUCTION}

The 2016 Nobel Prize awarded to Thouless, Haldane, and Kosterlitz has set an exclamation mark for the significance of topology in condensed matter physics [1]. Even before, the field of topology caught worldwide attention in 1985 when Klaus von Klitzing received the Nobel Prize for the discovery of the quantum Hall effect. The research field of topology continues to expand to various areas of physics. Topological insulators have been demonstrated to exist in several two- and three-dimensional materials [2-7]. Topological phases were also realized in a large and increasing variety of physical systems such as cold atoms in optical lattices [8], photonic Floquet crystals [9], and polaronic [10], acoustic [11], and mechanical systems [12,13]. A detailed understanding of topological materials is also of great interest for the application of electronic devices because they promise to allow one to reduce energy consumption by suppressing unwanted scattering.

Recently, quantum magnets have received much attention, in particular magnetically ordered systems [14-19]. But also a disordered valence bond crystal in a dimerized quantum magnet has displayed topologically non-trivial behavior [20-22]. Still, the number of established compounds displaying topologically non-trivial magnetic excitations is extremely limited and therefore novel topological compounds are of great interest. In particular, no candidate for a magnetic system displaying a quantized Zak phase in one dimension has been put forward.

Hence, the first of two key goals of the present article is to provide evidence for the existence of such a non-trivial topological phase in $\mathrm{BiCu}_{2} \mathrm{PO}_{6}$, which represents a quasione-dimensional (1D) quantum antiferromagnet [23-25]. The second goal is a general one reaching beyond the particular

\footnotetext{
*maik.malki@tu-dortmund.de
}

Published by the American Physical Society under the terms of the Creative Commons Attribution 4.0 International license. Further distribution of this work must maintain attribution to the author(s) and the published article's title, journal citation, and DOI. material $\mathrm{BiCu}_{2} \mathrm{PO}_{6}$; it concerns the existence of localized edge states in bosonic systems or fermionic bands away from the Fermi level. Often, the bulk-boundary correspondence is interpreted such that topological non-trivial invariants imply the existence of localized modes. We show that for the localization of edge modes generically the existence of an indirect gap, i.e., a finite energy difference independent of momentum, is necessary. In contrast, the existence of a nontrivial topology in a particular band only requires this band to be separated; i.e., the existence of a direct gap at each momentum is sufficient.

Of course, the significance of the energy gap for the localization of the edge modes is known and established; see for instance Refs. [26-30]. But since so far fermionic topological insulators have been the focus of research, it is natural to consider a filled valence and an empty conduction band separated by an energy gap with the Fermi level lying within this gap, i.e., a standard band insulator. This implies that the direct gap $\Delta_{\text {dir }}$ with momentum conservation and the indirect gap $\Delta_{\text {indir }}$ without momentum conservation are both finite. Very often the situation $\Delta_{\text {dir }}=\Delta_{\text {indir }}=\Delta$ is considered anyway and the generic length scale is inversely proportional to $\Delta$. The situation where $\Delta_{\text {dir }} \neq \Delta_{\text {indir }}$ holds has received little attention. In particular the situation with $\Delta_{\text {indir }}<0$ leading to self-doping has not been explored.

The situation is richer if one considers fermionic bands away from the Fermi energy or bosonic bands. These bands, or more precisely the corresponding eigenstates, can display well-defined non-trivial topologies if the bands are energetically separated from each other, i.e., if there are finite direct gaps at each momentum between the bands. But it is one of the goals of the present article to illustrate that this condition is not sufficient to ensure localized edge states: finite indirect gaps to the adjacent bands are required to protect the localization of the edge states.

Since $\mathrm{BiCu}_{2} \mathrm{PO}_{6}$ is essentially a one-dimensional system, the Berry phase qualifying for a topological invariant is the Zak phase $\Omega$ [31]. It can take any value between 0 and $2 \pi[\Omega \in[0,2 \pi)]$ because it measures the scalar product $\exp (\mathbf{i} \Omega)=\langle 2 \mid 1\rangle$ between a quantum state $|1\rangle$ at wave vector 0 and the quantum state $|2\rangle$ taken to wave vector $2 \pi$ by parallel 


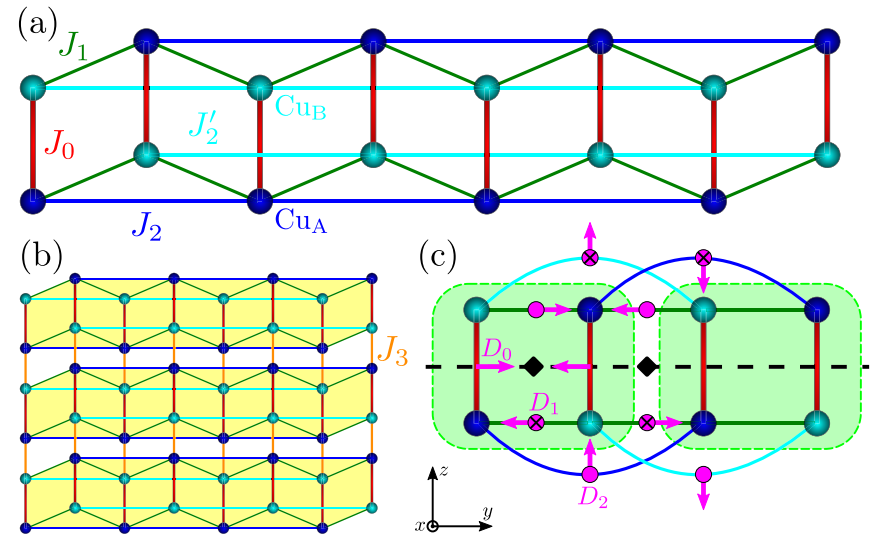

FIG. 1. Spin model of $\mathrm{BiCu}_{2} \mathrm{PO}_{6}$. (a) The tubelike structure realizes $1 \mathrm{D}$ frustrated spin ladders with two copper sites $\mathrm{Cu}_{\mathrm{A}}$ and $\mathrm{Cu}_{\mathrm{B}}$. The different bonds stand for different isotropic Heisenberg couplings. In the case of the minimal model the difference of copper sites is neglected so that $J_{2}=J_{2}^{\prime}$ holds. (b) Interladder isotropic Heisenberg coupling $J_{3}$ between adjacent spin ladders forming a weakly coupled two-dimensional (2D) system. (c) DM couplings of $\mathrm{BiCu}_{2} \mathrm{PO}_{6}$. Short violet arrows display the orientation of the DM vectors in $\mathbf{D}_{i j}\left(\mathbf{S}_{i} \times \mathbf{S}_{j}\right)$ where the sites $i$ and $j$ are ordered with ascending $y$ or $z$ coordinate; their length is not to scale. Inversion symmetry exists about the centers (black diamonds) of the plaquettes; reflection about the black dashed center line is a symmetry of the isolated, isotropic spin ladder.

transport. For inversion-symmetric systems the direction of parallel transport does not matter so that $\langle 1 \mid 2\rangle=\langle 2 \mid 1\rangle$ holds and $\Omega$ can be either 0 or $\pi$. Importantly, the Zak phase has been related to edge modes in strips of graphene [32]. It has been measured in systems of ultracold atoms in 1D optical lattices [33] and in twisted photons [34]. A related but different topological invariant is the winding number, which is discussed in Appendix F.

\section{MODEL FOR BiCu $\mathrm{PO}_{6}$}

The compound $\mathrm{BiCu}_{2} \mathrm{PO}_{6}$ is a low-dimensional quantum antiferromagnet of which the ground state is a valence bond solid. It does not show magnetic order, but it has a finite spin gap and a finite spin-spin correlation length. The spins are coupled antiferromagnetically in dimers interacting via further couplings [23-25,35,36]. The coupled dimers form a tubelike, frustrated spin-1/2 Heisenberg ladder as shown in Fig. 1(a). There are two types of copper ions $\mathrm{Cu}_{\mathrm{A}}$ and $\mathrm{Cu}_{\mathrm{B}}$ alternating along the ladders due to differing positions of the surrounding bismuth ions [35] (not shown here). The 1D spin ladders form stacked layers with weak but still measurable interladder couplings in each layer; see Fig. 1(b). The couplings between layers are negligible [36]. The dominating couplings are those along the spin ladders. The large atomic number $(Z=83)$ of bismuth induces an extraordinarily strong spin-orbit coupling so that the resulting magnetic exchange coupling is anisotropic with an important antisymmetric Dzyaloshinskii-Moriya (DM) coupling D [37] and concomitant symmetric coupling $\Gamma[38,39]$. The Hamilton operator reads

$$
\mathcal{H}=\sum_{i<j}\left[J_{i j} \mathbf{S}_{i} \cdot \mathbf{S}_{j}+\mathbf{D}_{i j} \cdot\left(\mathbf{S}_{i} \times \mathbf{S}_{j}\right)+\sum_{\alpha, \beta} \Gamma_{i j}^{\alpha \beta} S_{i}^{\alpha} S_{j}^{\beta}\right],
$$

where bold symbols represent vectors and $\mathbf{S}$ the spin vector operator. The couplings $J_{i j}$ are equal to $J_{0}, J_{1}, J_{2}$, and $J_{3}$ as shown in Fig. 1(a); $J_{0}$ is the dominating intradimer coupling while $J_{3}$ describes the interladder coupling. The intraladder couplings $J_{1}$ and $J_{2}$ or $J_{2}^{\prime}$ are the nearest neighbor and nextnearest neighbor interdimer couplings.

The elementary excitations of the spin isotropic ladder are dispersive triplons, i.e., hard-core $S=1$ quasiparticles [40], with the Hamiltonian without further interactions

$$
\mathcal{H}^{\text {iso. ladder }}=\sum_{k, \alpha} \omega_{0}(k) t_{k}^{\alpha, \dagger} t_{k}^{\alpha},
$$

where $t_{k}^{\alpha, \dagger}$ creates and $t_{k}^{\alpha}$ annihilates a triplon with wave vector $k$ and flavor $\alpha \in\{x, y, z\}$ [41]. The dispersion $\omega_{0}(k)$ is determined systematically by continuous unitary transformations in real space [42] and Fourier transformation.

The basic concept of continuous unitary transformations (CUTs) is to simplify a complex initial Hamiltonian $\mathcal{H}^{\text {init }}$ by applying continuous unitary transformations, i.e., gradual basis changes [43-45]. This goal is achieved by setting up a set of differential flow equations with renormalizing properties for running coupling constants. We use this approach to systematically derive Hamiltonians expressed in terms of creation and annihilation operators of elementary excitations, here triplons. For further details on the used CUT method we refer to Appendix B.

This isotropic model leads to a sixfold-degenerate triplon spectrum with six modes due to spin degeneracy and two dimers per unit cell which is at odds with experiment. This discrepancy with the experimental data emphasizes the importance of the DM terms breaking the spin isotropy. In order to include spin-anisotropic terms and the interladder terms we transform not only the isotropic Hamiltonian from the representation by spin operators to the representation by triplon operators, but also the spin operators. This allows us to express also the remaining parts of the Hamiltonian, the DM couplings and the interladder couplings, in terms of triplon operators. The resulting expressions are normal-ordered and the bilinear terms are kept, yielding a mean-field description of the elementary magnetic excitations of $\mathrm{BiCu}_{2} \mathrm{PO}_{6}$. $\mathrm{A}$ discussion of trilinear and quadrilinear interaction terms is provided below.

The anisotropic couplings are vital for lifting the spin degeneracy of the triplons [23-25]. It is established that the antisymmetric DM and the symmetric $\Gamma$ coupling have to be considered together $[38,39]$. In leading order, we use

$$
\Gamma_{i j}^{\alpha \beta}=\frac{D_{i j}^{\alpha} D_{i j}^{\beta}}{2 J_{i j}}-\frac{\delta^{\alpha \beta}\left(\mathbf{D}_{i j}\right)^{2}}{6 J_{i j}},
$$

which results from deriving the anisotropic exchange from a Hubbard model with spin-orbit coupling. The parametrization is chosen such that $\Gamma_{i j}^{\alpha \beta}$ does not comprise an isotropic component because it is included in the Heisenberg coupling $J_{i j}$. 


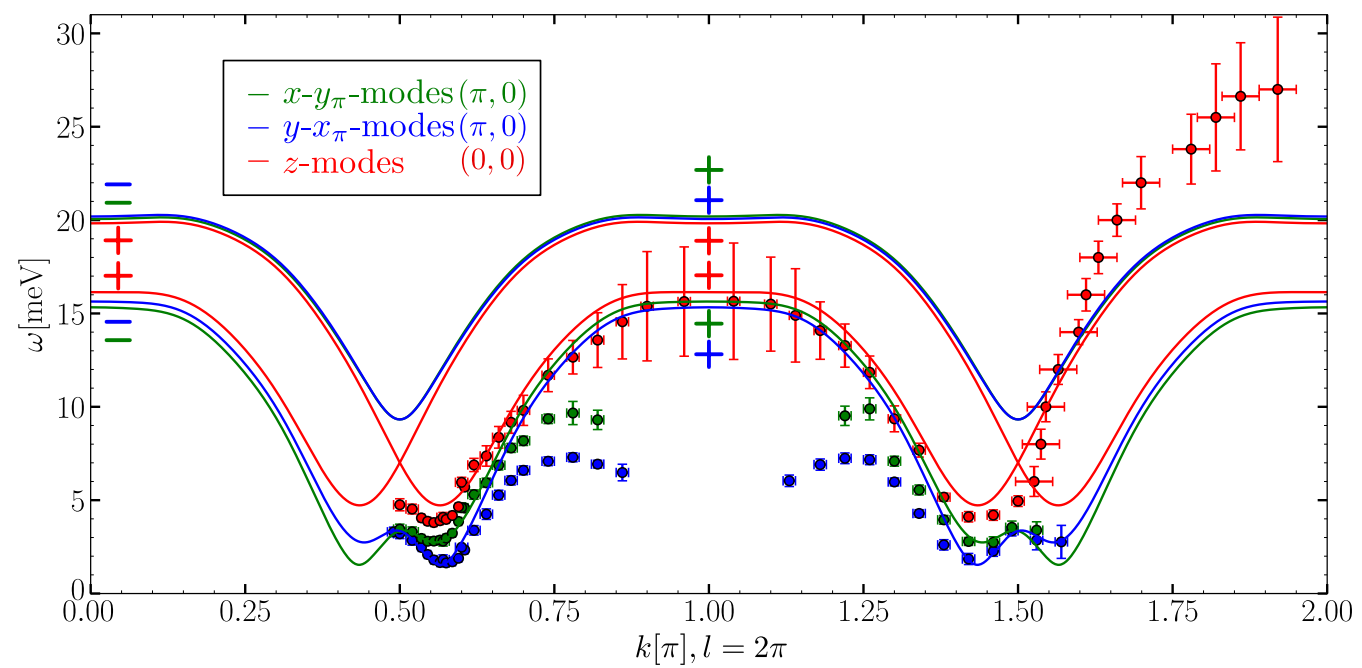

FIG. 2. Best fit of the one-triplon dispersions obtained for $J_{0}=9.4 \mathrm{meV}, J_{3}=1.5 \mathrm{meV}, J_{1}=1.2 J_{0}, J_{2}=1.09 J_{0}, D_{1}^{x}=0.58 J_{0}, D_{1}^{y}=$ $0.73 J_{0}, D_{2, a}^{z}=-0.02 J_{0}$, and $D_{3}^{y}=0.02 J_{0}$. Components not listed are zero. The symbols with error bars show the INS data [23]. The legend denotes the Zak phases of the modes in the $k$ and $l$ directions; see main text. The plus and minus signs at the time-reversal invariant wave numbers 0 and $\pi$ indicate the parities of the modes.

The possible directions of the DM vectors are constrained by the point group symmetries of the lattice; see Appendix A. The symmetry of $\mathrm{BiCu}_{2} \mathrm{PO}_{6}$ is higher if we neglect the difference between the two copper sites, see Fig. 1, dealing with a slightly simplified model which we call the minimal model [24]. In this minimal model, the DM vectors can have components as shown in Fig. 1(c) compatible with the Moriya symmetry rules [37]. If the difference between the $\mathrm{Cu}$ sites is taken into account the symmetry is reduced [35]; the possible DM vectors are given in Appendix A. But the additionally possible DM components are rather small because the copper sites are not very different electronically. Hence, we focus on the minimal model in the sequel.

The complete bilinear triplon Hamiltonian in momentum space is represented in a generalized Nambu notation (up to unimportant constants)

$$
\mathcal{H}=\frac{1}{2} \sum_{k, l} \mathbf{a}_{k, l}^{\dagger} \mathcal{M}_{k, l} \mathbf{a}_{k, l},
$$

where the wave number $k$ corresponds to the direction along the ladders while $l$ corresponds to the perpendicular direction; see Fig. 1(b). For brevity of notation, we combined the bosonic triplon operators into a column vector

$$
\mathbf{a}_{k, l}=\left(\mathbf{t}_{k, l}^{\top}, \mathbf{t}_{k+\pi, l}^{\top}, \mathbf{t}_{-k,-l}^{\dagger}, \mathbf{t}_{-k-\pi,-l}^{\dagger}\right)^{\top},
$$

with 12 components. Each boldface symbol stands for the three-dimensional vector $\mathbf{t}_{k, l}=\left(t_{k, l}^{x}, t_{k, l}^{y}, t_{k, l}^{z}\right)^{\top}$ and $\mathbf{t}_{k, l}^{\dagger}=$ $\left(t_{k, l}^{x, \dagger}, t_{k, l}^{y, \dagger}, t_{k, l}^{z, \dagger}\right)$. In this way, the Hamiltonian is described by a Hermitian $12 \times 12$ matrix

$$
\mathcal{M}_{k, l}=\left[\begin{array}{cc}
A(k, l) & B(k, l) \\
B^{\dagger}(k, l) & A^{\top}(-k,-l)
\end{array}\right],
$$

where the matrices $A(k, l)=A^{\dagger}(k, l)$ and $B(k, l)=$ $B^{\top}(-k,-l)$ are $6 \times 6$ matrices. Note that $\mathbf{a}_{k, l}$ and thus $\mathcal{M}_{k, l}$ are modified relative to Ref. [46] in order to consider momentum conservation. Further simplifications are discussed in Appendix B.

The eigenenergies and eigenmodes are obtained by a bosonic Bogoliubov transformation [46] leading to $\mathcal{H}=$ $\sum_{n, k, l} \omega_{n}(k, l) b_{n, k, l}^{\dagger} b_{n, k, l}$ where the index $n$ labels the six different modes at given wave vector $(k, l)$. The Bogoliubov transformation is based on usual bosons without the hardcore property. This is justified because the essential hard-core effects are considered in the transformation of the isolated spin ladder [24,42]. The one-triplon dispersions $\omega_{n}(k, l)$ are used to fit the inelastic neutron scattering (INS) data by adjusting the couplings $\left(J_{0}, D_{i j}^{\alpha}\right)$, keeping the ratios $J_{1} / J_{0}=1.2$, $J_{2} / J_{1}=0.9$, and $J_{3} / J_{0}=0.16$ fixed because these ratios are determined by the experimentally determined wave number $k$ where the gap $\Delta$ occurs and by the ratio between the measured lower maximum $\omega(k=\pi, l=2 \pi)$ and the gap $\Delta$ of the $z$ mode [24].

The dispersions in the $k$ direction in Fig. 2 agree very well with the experimental data at low energies. But there are discrepancies at higher energies, in particular the downturns of the modes given by the blue and green symbols. These features are explained qualitatively by the hybridization of the single modes with two-triplon continua, i.e., by decay processes [23] due to trilinear triplon terms in the Hamiltonian. But a quantitative description is not available so that the downturning modes can also stem from bound states, i.e., related to quadrilinear terms in the Hamiltonian. Plumb et al. suggest that the quasiparticle picture breaks down, but in view of the lack of a quantitative explanation of the downturns this does not need to be the case.

The downturns are a caveat of the single-particle model. Still, the description on this level can be justified by the following arguments.

(i) The energetically low-lying bosonic modes are protected by the finite energy gap so that perturbation theory is valid. Hence any coupling not considered in the single-particle description will only distort the states 
continuously so that discrete topological invariants will not be altered.

(ii) The topological twist causing the finite Zak phase computed below stems essentially from the region in $k$ space, namely the vicinity of $k=\pi / 2$ and $3 \pi / 2$, see Appendix E, where the single-bosonic modes are energetically low lying, well defined, and provide a reliable description of the experimental data without trilinear or quadrilinear terms in the Hamiltonian.

In addition, the Zak phase is computed in two ways, namely by direct computation of the phases of quantum states and by consideration of the parity of states with respect to inversion. Of course, both approaches are not unrelated because they rely on the same states in the single-particle model.

In conclusion, due to the lack of an established and tractable model beyond the bilinear level, our results provide evidence but not ultimate proof of the existence of a finite Zak phase. We point out that the situation is similar in many analyses of electronic solid state systems where effective lowenergy models are used for the determination of topological invariants. These models are justified in the vicinity of certain points in the Brillouin zone, but do not describe the whole dispersion and the evolution of eigenstates through the entire Brillouin zone; see for instance Refs. [2,30,47,48]. The use of the concept of electronic quasiparticles is only justified in Fermi liquid theory at low energies while the dynamics at higher energies is dominated by continua.

\section{BERRY PHASES IN BOSONIC SYSTEMS}

To assess the topological properties of bosonic bands the Berry connection needs to be generalized to bosonic systems. Even for non-interacting bosons this is not trivial. While for fermions the scalar product of quantum states can be naturally transferred to fermionic operators because a fermionic Bogoliubov transformation is unitary this does not hold for bosonic operators and transformations. For bosonic systems a symplectic product $\left\langle\left\langle\mathbf{k}_{1}, n_{1} \mid \mathbf{k}_{2}, n_{2}\right\rangle\right\rangle$ is required. We provide a suitable definition for the symplectic product in Appendix C. Based on this symplectic product the standard relations [5] for the Berry connection

$$
\mathcal{A}_{n, \text { sym }}(\mathbf{k})=\mathbf{i}\left\langle\left\langle\mathbf{k}, n\left|\nabla_{\mathbf{k}}\right| \mathbf{k}, n\right\rangle\right\rangle
$$

and the Berry phase

$$
\Omega_{n}=\oint \mathcal{A}_{n}(\mathbf{k}) d \mathbf{k}=\mathbf{i} \oint\left\langle\left\langle\mathbf{k}, n\left|\nabla_{\mathbf{k}}\right| \mathbf{k}, n\right\rangle\right\rangle d \mathbf{k}
$$

can be kept. If the closed path in the above equation encompasses the Brillouin zone, $\Omega_{n} /(2 \pi)$ defines the Chern number. The Chern number in $\mathrm{BiCu}_{2} \mathrm{PO}_{6}$ remains trivially zero even if magnetic fields are included because the material is essentially one-dimensional.

The characteristic Berry phase for one-dimensional systems is the Zak phase [31,32]. It is computed in one direction in the periodic Brillouin zone [31]. Due to the periodicity in $k$ and $l$ space the closed loops $k \rightarrow k+2 \pi$ or $l \rightarrow l+2 \pi$ both allow us to define Berry phases. They can be averaged over the respective other wave vector yielding the vector $\mathbf{P}$ [49]:

$$
\mathbf{P}=\frac{1}{2 \pi} \int \mathcal{A}_{n, \mathrm{sym}} d k d l .
$$

Its value for each triplon band is given in the legend of Fig. 2. The $z$ mode remains topologically trivial $\mathbf{P}=(0,0)$ while the coupled $x-y_{\pi}$ and $x_{\pi}-y$ modes display the Zak phase $\mathbf{P}=(\pi, 0)$; for computational technicalities see Appendix D. A non-trivial phase occurs in the ladder direction, but not perpendicular to it.

One may worry that the dispersion branches of $x-y_{\pi}$ and $y-x_{\pi}$ modes are not separated, and even intersect at $k=\pi / 2$ and $k=3 \pi / 2$. These intersections are clear crossings so that one can track the states easily by following the differentiably evolving dispersions. As a result, the Zak phases are well defined. In addition, we studied what happens if a small perturbation, here a magnetic field, lifts the degeneracy at the intersections and converts the crossings to anticrossings. But since the intersecting modes all have the same Zak phase this braiding is trivial and does not make a difference. Thus the Zak phases are robust and remain unaltered so that the topology is unchanged.

The $l$ dependence in the investigated minimal model mainly enters via the isotropic term $J_{3} \cos (l)$, which does not alter the eigenmodes since this term is proportional to unity. The small $D_{3}^{y}$ and the even smaller $\Gamma_{3}^{\alpha \alpha}$ barely have an impact on the dispersion and on the eigenmodes. We checked that even larger values of $D_{3}^{y}$ and $\Gamma_{3}^{\alpha \alpha}$ do not induce a non-trivial Zak phase in the $l$ direction or a finite Chern number. So they do not influence the topology.

The Zak phase is constant for all values of $l$ being either zero or $\pi$. It is pinned to these particular values in $\mathrm{BiCu}_{2} \mathrm{PO}_{6}$ because the system is inversion symmetric; see Fig. 1(c). The transformation operator of inversion is given by the matrix $\Pi=\operatorname{diag}(1,1,1,-1,-1,-1,1,1,1,-1,-1,-1)$ with $\Pi^{2}=\mathbb{1}$ which transforms $\Pi \mathcal{M}_{k, l} \Pi=\mathcal{M}_{-k,-l}$ and hence ensures the quantization of the Zak phase as multiples of $\pi$.

We stress that the Zak phase is robust; i.e., small changes of the model do not alter it. For instance, it remains the same if we pass from the minimal model to the extended model accounting for different copper sites. Similarly, one may reduce the values of $D_{1}^{x}$ and $D_{1}^{y}$ even by a factor 2 , cf. Ref. [23], and still obtain the same Zak phase. Moreover, the substantial twist leading to the non-trivial Zak phase stems from the vicinity of the minima of the dispersions; i.e., the low-lying modes matter most. It is crucial that the DM components $D_{1}^{x} \neq D_{1}^{y}$ are different in order to have a non-trivial Zak phase. If they are equal, the topological bands are no longer separated so that either no Zak phase can be defined or it is trivial. But $D_{1}^{x} \neq D_{1}^{y}$ is required in order to fit the experimental data. This becomes clear upon analyzing the effects of $D_{1}^{x}$ and $D_{1}^{y}$ mediated by their symmetric couplings $\Gamma_{1}^{x x}$ and $\Gamma_{1}^{y y}$ on the dispersion [24]. If both parameters were equal, the corresponding dispersions would be the same as well. This is the crucial point: the experimental data show that the two lowest-lying modes differ. Hence the values of the components $D_{1}^{x}$ and $D_{1}^{y}$ must be different. Furthermore, the quantized Zak phase persists in the presence of applied magnetic fields which do not close the spin gap above the ground state. This insensitivity results from the fact that the 

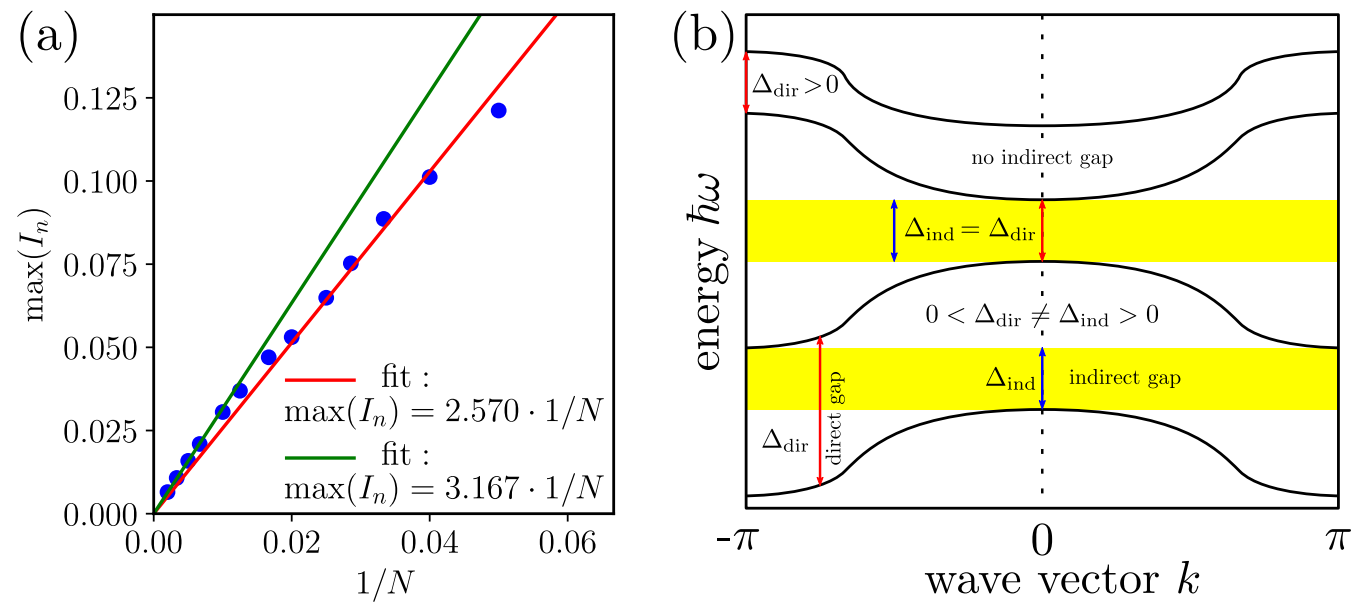

FIG. 3. Panel (a): Extrapolation of the largest IPR of a finite piece of chain in the minimal model for $\mathrm{BiCu}_{2} \mathrm{PO}_{6}$ clearly indicating that all states are extended, no localized edge states are present. Panel (b): Sketch of a direct gap $\Delta_{\text {dir }}$ at the red arrows and of an indirect gap $\Delta_{\text {ind }}$ (blue arrows). A direct gap is the minimal difference between the maximal energy of a lower mode and the minimal energy of an upper mode at the same momentum. In contrast, the indirect gap is given by a forbidden energy interval (shown in yellow) between two modes irrespective of momentum conservation.

Zak phase is generated by the coupling between the $k$ and $k+\pi$ wave vectors. Thus, terms coupling at the same wave vectors such as uniform magnetic fields do not destroy it. As mentioned above, even lifted crossings do not change the Zak phases.

The wave vectors with $k, l \in\{0, \pi\}$ are invariant under inversion so that states at these values have a well-defined inversion parity denoted by "+" and by "_" in Fig. 2. The product of the parities at $k=0$ and $k=\pi$, both at $l=2 \pi$, equals the exponential of the corresponding Zak phase [50] in perfect agreement with the direct computation of the Zak phase in the $k$ direction. This alternative route to the Zak phase adds another piece of evidence that $\mathrm{BiCu}_{2} \mathrm{PO}_{6}$ is a low-dimensional quantum magnet with a finite non-trivial Zak phase, but it is not unrelated to the direct calculation of the Zak phase because it relies also on the eigenstates of the same model.

\section{IV. (NON) EXISTENCE OF LOCALIZED EDGE STATES}

We are looking for a smoking gun signature of a non-trivial Zak phase. The important bulk-boundary correspondence [5] in its conventional interpretation states that at the boundaries between topologically non-trivial and trivial phases localized states must exist closing the gaps between the topologically distinct bands. Otherwise, the abrupt change of discrete topological invariants cannot be accounted for. These so-called edge states should exist at the ends of strips with a finite discrete Zak phase or a finite winding number [32,51,52] in the bulk. Hence, we are looking for localized edge states at the ends of finite strips of the minimal model for $\mathrm{BiCu}_{2} \mathrm{PO}_{6}$. But we have not found any localized edge states; see below.

We studied the localization of all states quantitatively by computing the inverse participation ratio (IPR) [53,54], which is a standard measure for localization. To this end, we calculate the eigenstates of a finite piece of chain with open boundaries so that the IPR can be determined for each of them. If the IPR tends to zero for increasing system size the state is extended; if it stays finite the corresponding state is localized. We use the definition

$$
I_{n}=\sum_{i} p_{n, i}^{2}=\sum_{i}|\langle\langle n, i \mid n, i\rangle\rangle|^{2}
$$

adapted to the bosonic symplectic product and find that all $I_{n} \rightarrow 0$. This is shown by studying the largest IPR in a finite chain segment. Figure 3(a) extrapolates this largest IPR by a linear function of the inverse system size. Obviously, the largest IPR decreases monotonically to zero and hence all IPRs vanish in the thermodynamic limit.

This puzzling fact can be explained by three arguments. First, the usual argument of bulk-boundary correspondence [5] requires the existence of states within the band gaps of the topologically non-trivial bulk systems; let us call them in-gap states [55]. (In the literature, these states are often called midgap states if a chiral symmetry enforces them to lie at zero energy.) The localization of these states is warranted if they lie within an energy gap [26-29,32]. But which gap matters is less obvious and is the subject of our study.

Second, the energy of most localized edge states lies in an indirect gap; i.e., independently of momentum conservation they are protected by energy conservation. For an illustration of direct and indirect gaps see Fig. 3(b). If there is an indirect gap the energetic protection persists even if we sum over all wave vectors, for instance to compute local densities of states. In contrast, in the absence of an indirect gap the energetic separation of the in-gap states from the bulk states is only ensured by the conserved momentum $\hbar k$. We stress that introducing boundaries, for instance in the $y$ direction, lifts the conservation of momentum so that generically the momenta hybridize and the in-gap states mix with scattering states in the continua and thereby the in-gap states delocalize. Even at this stage, one may wonder whether the in-gap states are localized or not. If the boundary had only the effect to reflect the incident triplon it would only mix $k$ and $-k$ and a direct energy gap would still be sufficient to induce localization. Yet this is not the case in $\mathrm{BiCu}_{2} \mathrm{PO}_{6}$ where the bands 


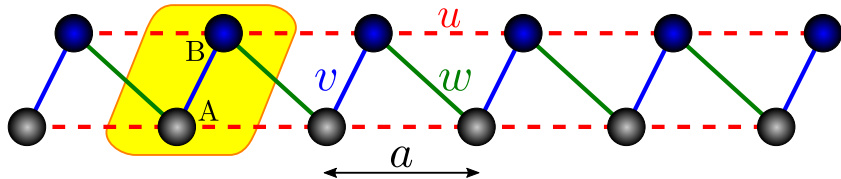

FIG. 4. Lattice of the extended SSH model with intracell coupling $v$, intercell coupling $w$ between nearest neighbors, and intercell coupling $u$ between next-nearest neighbors. The unit cell comprising sites A and B is displayed by the yellow area.

are separated by direct gaps, but not by indirect gaps. It is known that this mechanism weakens localization in ordinary quantum systems [56,57] and the localization length has been linked to the precise momentum dependence of the dispersion in topological insulators with direct gaps [28]. Recently, it has been shown that localization of edge states may persist in a topological system if the hybridization is sufficiently suppressed by various symmetries [58,59] or if the effective surface Hamiltonian continues to have non-trivial topological invariants [30]. We emphasize, however, that the outstanding significance of indirect gaps has not been mentioned in the literature.

The third argument resides in the independence of the topological invariants in the bulk of the eigenenergies. The vector potential (7) and hence the Berry phase (8) depend on the eigenmodes only. But they are blind to their eigenenergies, i.e., to the dispersions. Thus, one can modify the Hamiltonian leaving the eigenmodes and the topology completely unaltered while shifting the dispersion energies arbitrarily. This argument has been put forward for strips of graphene and transversal dispersions in weakly coupled Su-SchriefferHeeger models [32], but not yet for parallel dispersions. Such changes have an effect on the edge modes if boundaries are introduced [55]. We illustrate this point in an extended $\mathrm{Su}-$ Schrieffer-Heeger model.

\section{V. (DE)LOCALIZATION IN THE SU-SCHRIEFFER-HEEGER MODEL}

Since the topology of bosonic systems is still less known and the model for $\mathrm{BiCu}_{2} \mathrm{PO}_{6}$ is rather intricate so that certain details may hide the relevant aspects, we want to support our hypothesis on the delocalization of edge states by a transparent calculation for an established and well-known fermionic model, the Su-Schrieffer-Heeger (SSH) model [32,60]. In this paradigmatic model, we show explicitly that adding a coupling, which does not alter the eigenstates, can delocalize the edge modes. If the indirect gap vanishes, the edge modes become extended; i.e., they are no longer modes at the edge in the proper sense of the word.

We consider the SSH model and extend it slightly by the coupling $u$ between next-nearest neighbors; see Fig. 4. Its Hamiltonian reads

$$
\begin{aligned}
\mathcal{H}_{\mathrm{SSH}}= & \sum_{i}^{N}\left(v c_{i, \mathrm{~B}}^{\dagger} c_{i, \mathrm{~A}}+w c_{i+1, \mathrm{~A}}^{\dagger} c_{i, \mathrm{~B}}\right. \\
& \left.+u c_{i+1, \mathrm{~A}}^{\dagger} c_{i, \mathrm{~A}}+u c_{i+1, \mathrm{~B}}^{\dagger} c_{i, \mathrm{~B}}\right)+ \text { H.c. }
\end{aligned}
$$

where $c_{i, \mathrm{~A}}$ is the fermionic annihilation operator on site A of unit cell $i$ and $c_{i, \mathrm{~B}}$ the corresponding fermionic annihilation operator on site $\mathrm{B}$. The creation operators are the Hermitian conjugate ones. All three couplings are real and shown in Fig. 4. The Hamiltonian $\mathcal{H}_{\mathrm{SSH}}$ is particle conserving. Without the next-nearest neighbor coupling $u$, but with perpendicular couplings, the model was also studied by Delplace et al. [32].

In the bulk or for periodic boundary conditions Fourier transformation yields

$$
\begin{aligned}
\mathcal{H} & =\sum_{k}\left(c_{k, \mathrm{~A}}^{\dagger} c_{k, \mathrm{~B}}^{\dagger}\right) \mathcal{M}_{k}\left(\begin{array}{c}
c_{k, \mathrm{~A}} \\
c_{k, \mathrm{~B}}
\end{array}\right), \\
\mathcal{M}_{k} & =\left(\begin{array}{cc}
2 u \cos (k) & v+w e^{i k} \\
v+w e^{-i k} & 2 u \cos (k)
\end{array}\right),
\end{aligned}
$$

where the lattice constant $a$ is set to unity.

The ensuing dispersion is

$$
\varepsilon_{n}(k)=2 u \cos (k) \pm \sqrt{v^{2}+w^{2}+2 v w \cos (k)}
$$

with $n \in\{1,2\}$ corresponding to the \pm sign in front of the square root. The dispersion branches are depicted in the upper row of Fig. 5 for $v=0$ and the indicated ratios $u / w$.

On the one hand, the eigenstates are the same as in the usual SSH model without next-nearest neighbor coupling $u$ since the additional coupling leads to a modification of the matrix $\mathcal{M}_{k}$ proportional to the $2 \times 2$ identity matrix $2 u \cos (k) \mathbb{1}_{2}$. For this reason, we call the coupling $u$ isotropic. The induced modification does not change the eigenstates at all. Hence, the extended SSH model shows the same Zak phase and the same winding number as the non-extended SSH model.

On the other hand, however, the numerical analysis of a finite piece of chain with the open boundary condition reveals that the localization of the edge states is not protected against the isotropic coupling despite the fact that the direct gap does not close so that the two bands remain separated; see Fig. 5. By the naked eye one already discerns that the wave function with the largest value of the inverse participation ratio (IPR) defined in Eq. (10), see also Refs. [53,54], is localized if the energy of the edge modes lies well within the indirect gap. But upon decreasing the indirect gap to zero for $u \rightarrow w / 2$ the IPR also drops to zero in the thermodynamic limit $N \rightarrow \infty$. Then it is obvious that the corresponding states are no longer localized. We emphasize that this does not contradict the logic of the bulk-boundary correspondence which requires that the energy gap has to close at the boundary to another phase with a different quantized topological invariant [5].

The IPR is the standard measure of localization in perfect agreement with the computed eigenstates shown in the lower row of Fig. 5. But it may be argued that remnants of localization persist in local spectral properties in which many eigenstates contribute. The local density of states (LDOS) at the ends of finite chain pieces is a promising candidate to display fingerprints of localized edge states. Three representative results for the LDOS at the chain ends of the SSH model are displayed in Fig. 6. In the regime of finite indirect energy gaps where the IPR confirmed a localized edge state a clear resolution-limited peak, a $\delta$ peak, is found; see upper panel. But it is obvious that the delocalization of 

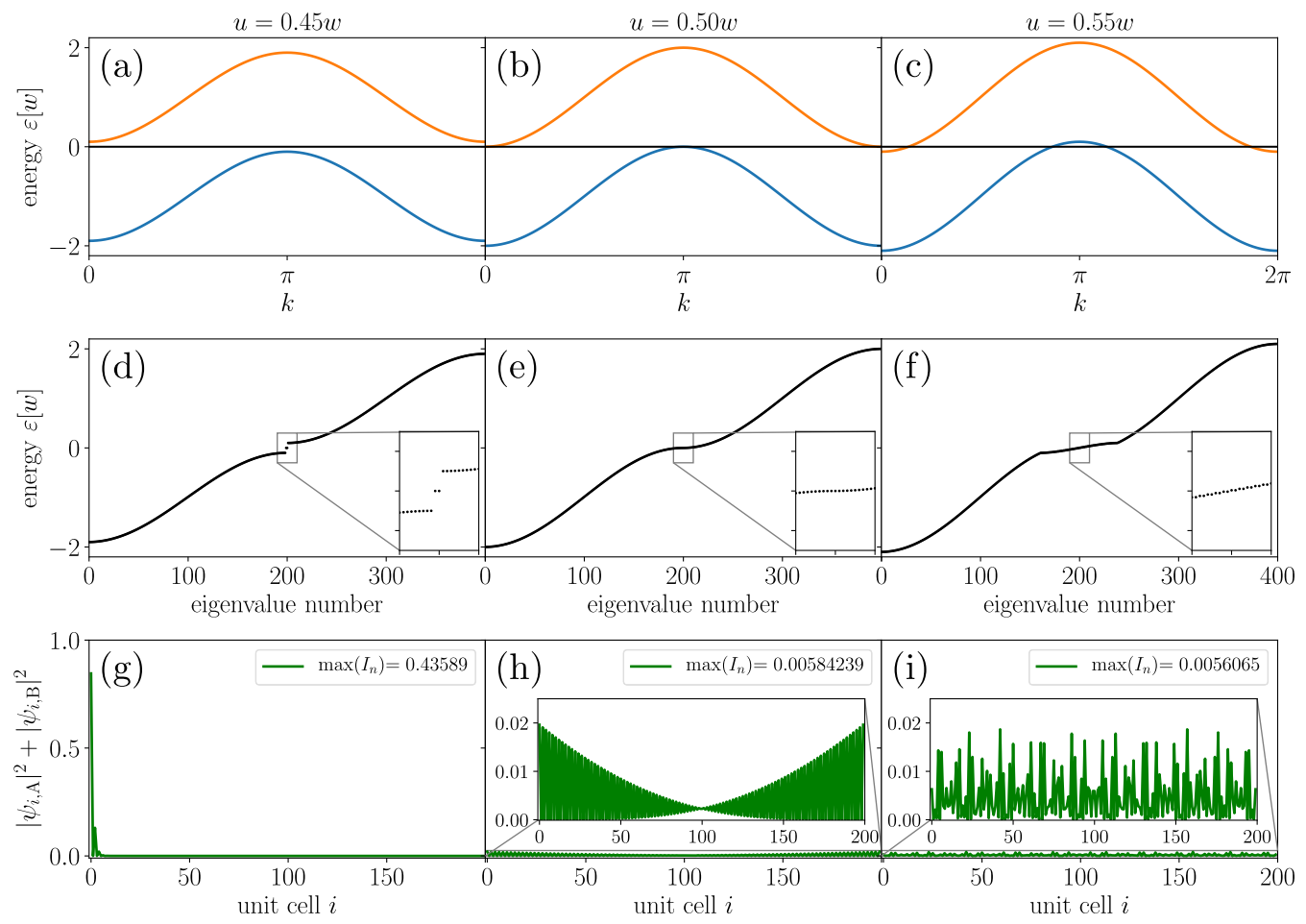

FIG. 5. Delocalization of the edge states illustrated for $v=0$. The three rows show, from top to bottom, the bulk dispersion, the eigenvalues of a finite piece of chain with open boundaries consisting of 200 unit cells, and the probability density $\left|\psi_{i, \mathrm{~A}}\right|^{2}+\left|\psi_{i, \mathrm{~B}}\right|^{2}$ of the eigenstate $\psi$ with the highest inverse participation ratio (IPR) given in the legend of the lower panels. The three columns refer to different ratios $u / w$. For $u=0.45 w$, the two edge modes lie within the indirect gap and are well-localized. The case $u=0.50 w$ is marginal and for $u=0.55 w$ no indirect gap exists anymore. Concomitantly, no localized modes exist. But the two bands continue to be clearly separated and hence well-defined.

this in-gap state is accompanied by the broadening of this $\delta$ peak which reduces to a diverging singularity in the marginal case $u=0.5 w$ where the indirect gap has just closed. In the

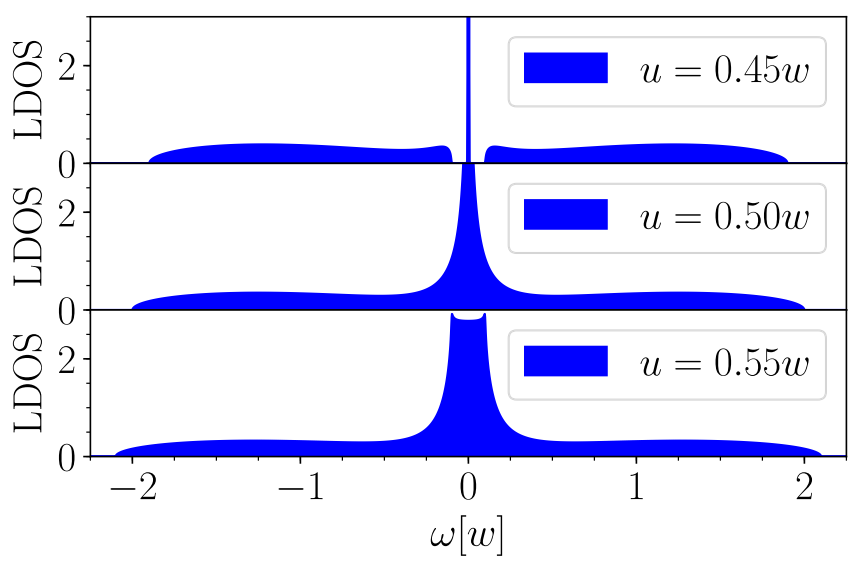

FIG. 6. Local density of states at one end of a finite piece of chain of the SSH model in (11). The upper panel displays the case where the indirect gap is still finite: a clear resolution-limited $\delta$ peak occurs at zero energy. The middle panel displays the marginal case where the indirect gap has just vanished. Clearly, no $\delta$ peak is present, but there is still a prominent peak which is no longer resolution limited; i.e., it has an intrinsic width. The lower panel displays the case where no indirect gap is present. Interestingly, a broad feature occurs, which is reminiscent of a one-dimensional miniband with slightly broadened Van Hove singularities at the edges. region where the indirect gap does not exist (or is negative, depending on the definition) no divergence occurs, but only a broad feature is present which is reminiscent of the density of states of a miniband due to its Van Hove singularities at the edges. Hence our conclusion is corroborated that the bulk-boundary correspondence does not imply localization. Peaklike structures may persist in local spectral quantities, but they differ qualitatively from the $\delta$ peak for positive indirect energy gaps.

\section{LOCAL DYNAMIC STRUCTURE FACTOR IN BiCu $2 \mathrm{PO}_{6}$}

In the preceding section, we illustrated and supported our scenario by an analysis of the (de)localization of the edge modes in the paradigmatic fermionic SSH model for simplicity and transparency. This analysis comprised the IPR and a local spectral property, the local density of states.

Here we return to the quantum magnet $\mathrm{BiCu}_{2} \mathrm{PO}_{6}$. The IPR has been analyzed and shown in Fig. 3(a). It is undoubtedly the suitable measure for the localization of a single state $[53,54]$. Yet we want to extend the analysis to local behavior in spectral properties. To this end, the local dynamic structure factor (local DSF) at the end of a spin ladder suggests itself. It displays a $\delta$ function if there is a localized edge state. It vanishes if the edge state delocalizes. But a certain residual peak made from many eigenstates could remain; see Sec. V on the SSH model. 

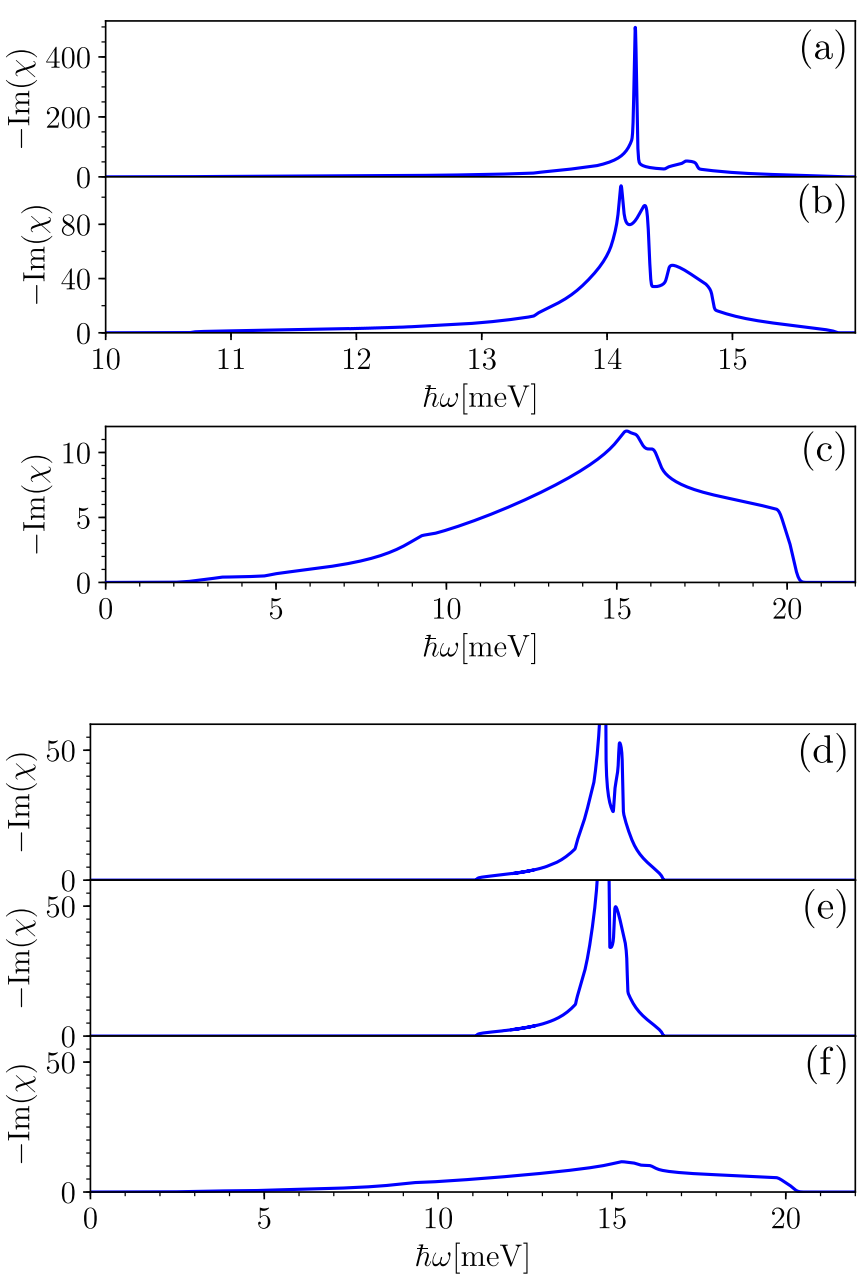

FIG. 7. The local dynamic structure factor (DSF) as a function of transferred energy. Panels (a) and (d) show the case of localized edge states while panels (b) and (e) show the marginal DSF for a closed indirect energy gap. Panels (c) and (f) correspond to the expected local DSF in $\mathrm{BiCu}_{2} \mathrm{PO}_{6}$ with negative indirect gap and without any traces of localization. The difference between panels (a)-(c) and panels (d)-(f) consists of the scales. The upper panels have adapted scales to show the details, in particular the $\delta$ peak, while the lower panels use the same scale and thus show the total evolution of the DSF.

To study potential spectral peaks, Fig. 7 displays the local DSF at the end of a finite piece of spin ladder with variations of the model for $\mathrm{BiCu}_{2} \mathrm{PO}_{6}$ with 1000 dimers, i.e., 1000 sites for triplons. At zero temperature, the DSF equals the imaginary part of the spin correlation function $\chi$. The result for the actual model for $\mathrm{BiCu}_{2} \mathrm{PO}_{6}$ is shown in panels (c) and (f). No sharp peak is discernible which could be related to a localized edge mode. But it is a priori unclear where such a peak should be and whether a less specific broad feature represents the remnant of localization; see Sec. V, on the SSH model. Thus, it is instructive to study the DSF of a modified model which has localized edge modes and which can be continuously tuned to the minimal model of $\mathrm{BiCu}_{2} \mathrm{PO}_{6}$.

To this end, we consider the following variation of the Hamiltonian. The dispersion of the isotropic spin ladder in
Eq. (B8) is replaced by

$$
\omega_{0}(k)=1.55+u \cos (k) .
$$

This choice leads to a finite indirect gap for which we expect localized edge modes. For the Hamiltonian with $u=0$, Fig. 7(a) shows the (resolution limited) $\delta$ peak as it results from the existing localized edge state. In contrast, setting $u=0.013554$ as in Fig. 7(b) leads to the marginal case of an indirect gap which has just vanished. Then, the DSF still displays a peak even though it is not a resolution-limited $\delta$ peak anymore. Clearly, it has acquired a certain intrinsic width. If the dispersion is changed further to the realistic one of the isotropic spin ladder so that $\mathrm{BiCu}_{2} \mathrm{PO}_{6}$ is described, all traces of a peak are completely gone; see Figs. 7(c) and 7(f). In order to be able to compare the various DSFs and to assess their evolution the three cases are shown in Figs. 7(d)-7(f) with the same scale.

In essence, we conclude that in $\mathrm{BiCu}_{2} \mathrm{PO}_{6}$ a quantized $\mathrm{Zak}$ phase is present, but no signatures of localized edge modes occur.

\section{CONCLUSIONS}

Two key results are presented in this article: One is material specific while the other is general.

The material-specific result is that we analyzed the inelastic neutron scattering data of $\mathrm{BiCu}_{2} \mathrm{PO}_{6}$ quantitatively on the basis of a model of non-interacting triplons. This model describes the low-lying magnetic modes very well. Discrepancies at higher energies may be attributed to decay and interaction processes. The topological twists, however, occur at low energies. Additional twists at high energies cannot be fully excluded. Due to the inversion symmetry of the system, the Zak phase is quantized in multiples of $\pi$. We found evidence that $\mathrm{BiCu}_{2} \mathrm{PO}_{6}$ is a disordered onedimensional quantum magnet displaying a finite, non-trivial Zak phase of $\pi$. This result was obtained by two, however related, approaches: (i) direct calculation of the Zak phase and (ii) the study of the parity of the modes at inversionsymmetric momenta. In addition, the computation of the winding number in Appendix F corroborates the conclusion of a non-trivial magnetic topology. The finite Zak phase is a robust result since it essentially depends on the difference of the Dzyaloshinskii-Moriya couplings $D_{1}^{x}$ and $D_{1}^{y}$.

The general result concerns the bulk-boundary correspondence which constitutes a fundamental concept in topological systems. We found no localized edge states in $\mathrm{BiCu}_{2} \mathrm{PO}_{6}$ in spite of the non-trivial Zak phase and the winding number. We explained this behavior by the delocalization of the edge modes due to a negative indirect energy gap between excitation modes while the direct gap remains positive so that the bands are well separated. Adding momentum-dependent terms to the Hamiltonian which are proportional to the identity does not change the topology in the bulk, but they strongly influence the localization in confined geometries. From this observation we deduced the generic importance of a positive indirect gap in order to have localized edge modes. This claim was supported by the analysis of an extended Su-SchriefferHeeger model. Meanwhile, we could extend our observation of the vital importance of a finite, positive indirect gap for 
the existence of localized edge modes in two-dimensional Chern insulators as well [55]. We view this scenario as generic because it occurs without particular interactions in typical, standard models without any fine-tuning.

We stress that delocalization due to a negative indirect gap is generic although in particular situations localization may be protected in spite of vanishing gaps. This is known from systems without topological properties [56,57] and from systems with topological properties where further symmetries, e.g., conservation of momentum and energy, can preserve localization although the edge state is coupled to gapless scattering states in the bulk [58,59]. Effective surface Hamiltonians can also display localized edge states despite coupling to topologically trivial bulk continua at certain special momenta [30].

The conceptual insight into the significance of a positive indirect gap beyond the condition of a positive direct gap has not been appreciated before. It underlines that localized edge states are protected by topology and by the structure of the spectrum, i.e., the existence of an indirect gap. While the direct gap protects the topological properties in the bulk, it is not sufficient to protect the localization of edge modes in confined geometries. Hence, the edge states are extended and cease to exist as edge states in the proper sense of the word implying localization at the edge. Still, they are in-gap states and as such in accordance with the derivation of the established bulk-boundary correspondence [5].

\section{ACKNOWLEDGMENTS}

We acknowledge useful discussions with Christoph $\mathrm{H}$. Redder and Joachim Stolze and provision of the experimental data by Kemp Plumb and Young-June Kim. Financial support (M.M.) was given by the Studienstiftung des Deutschen Volkes and by the Deutsche Forschungsgemeinschaft and the Russian Foundation of Basic Research through the transregio TRR 160. G.S.U. acknowledges the hospitality of the University of New South Wales during the finalization of the manuscript and the support of the Heinrich-Hertz Stiftung of North Rhine-Westphalia.

\section{APPENDIX A: SYMMETRY ANALYSIS OF BiCu $\mathrm{PO}_{6}$}

The direction of the Dzyaloshinskii-Moriya (DM) vectors $\mathbf{D}_{m}, m \in\{0,1,2,3\}$, is restricted due to the symmetries of the system. These restrictions are formulated by the five selection rules of Moriya [37] which relate the different couplings based on the point group symmetries of the system. For the sake of completeness, we present these five selection rules here briefly. Moriya established them by considering two interacting ions with spins whose positions we label with $A$ and $B$. The center of the connecting line $\overline{A B}$ is denoted by $C$.

First. If $C$ presents a center of inversion, then $\mathbf{D}=0$ holds.

Second. If there is a mirror plane perpendicular to $\overline{A B}$ and passing through $C$, then $\mathbf{D} \perp \overline{A B}$ is valid.

Third. If a mirror plane including the positions $A$ and $B$ is present, the vector $\mathbf{D}$ is perpendicular to this mirror plane.

Fourth. In case of a twofold rotation axis perpendicular to the line $\overline{A B}$ and passing through $C$, then $\mathbf{D}$ is perpendicular to this twofold rotation axis.
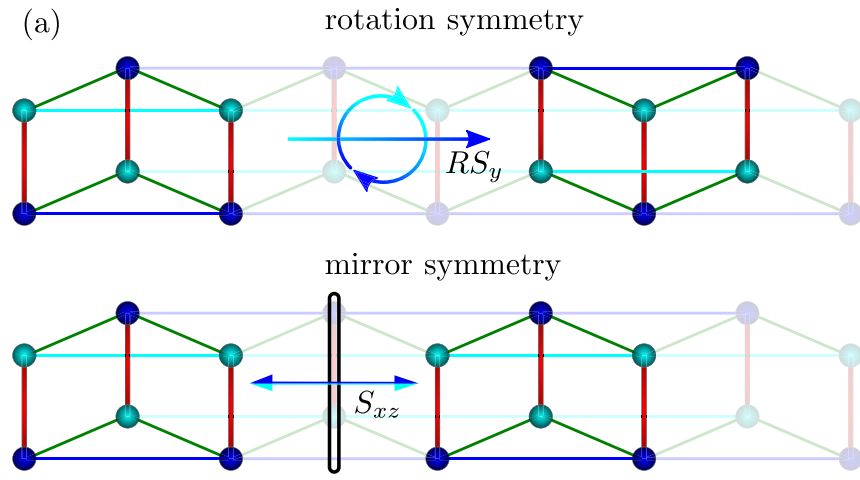

(b)
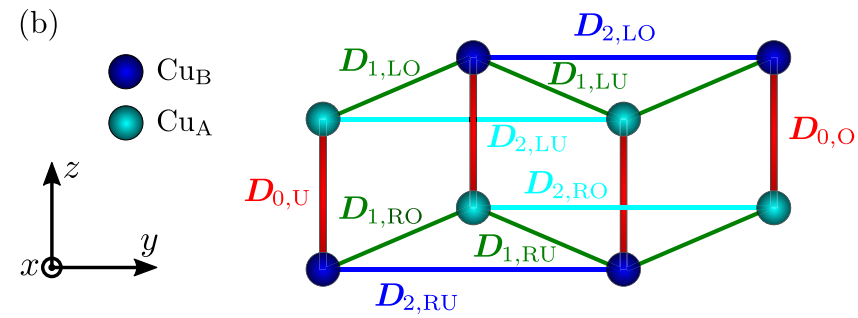

FIG. 8. Symmetry analysis. (a) Illustration of the two symmetry operations $R S_{y}$ and $S_{x z}$ leaving the model of $\mathrm{BiCu}_{2} \mathrm{PO}_{6}$ invariant. (b) Notation of the various $\mathbf{D}$ vectors in $\mathrm{BiCu}_{2} \mathrm{PO}_{6}$.

Fifth. If there is an $n$-fold axis $(n \geqslant 2)$ passing along $\overline{A B}$, the relation $\mathbf{D} \| \overline{A B}$ is valid.

Besides the information that specific $\mathbf{D}_{i j}$ components are forbidden due to point group symmetries of the single bonds one can additionally obtain information on the signs of the possible $\mathbf{D}_{i j}$ along the ladder by considering translations and glide reflections. Likewise the parity of the components relative to reflection about the center line, see Fig. 1(c) in the main article, can be elucidated. This parity determines whether a term contributes to the dispersions on the level of bilinear Hamiltonians or not [61].

If we neglect the difference between the two copper ions $\mathrm{Cu}_{\mathrm{A}}$ and $\mathrm{Cu}_{\mathrm{B}}$ we arrive at the minimal model of $\mathrm{BiCu}_{2} \mathrm{PO}_{6}$ with the possible DM components shown in Fig. 1(c) of the main article. Taking into account the difference between the two copper sites [35] the symmetry of the lattice is lower so that more $\mathbf{D}_{i j}$ components are allowed. Then only the following two symmetries of the crystal structure are present:

(1) $R S_{y}$ : Rotation by $\pi$ around the $y$ axis located in the middle of the spin ladder and a shift by half a unit cell.

(2) $S_{x z}$ : Reflection at the $x z$ plane located at a dimer.

These two symmetry operations are shown in Fig. 8(a). In Fig. 8(b) the notation of the various DM vectors is shown.

The determined symmetries imply the following constraints. The vector $\mathbf{D}_{0}$ only has a $y$ component due to the third selection rule based on the symmetry $S_{x z}$. The $R S_{y}$ symmetry yields the relation

$$
R S_{y}\left(\mathbf{D}_{0, \mathrm{U}}\right)=-\mathbf{D}_{0, \mathrm{O}}
$$

After an $R S_{y}$ rotation the stipulated sequence of the spin operators within the term $\mathbf{D}_{i j}\left(\mathbf{S}_{i} \times \mathbf{S}_{j}\right)$ (according to ascending $y$ and $z$ coordinate) must be recovered by swapping the spin operators. Thus, Eq. (A1) shows the alternating behavior of $D_{0}^{y}$ 
along the legs. The symmetry analysis of the $\mathbf{D}_{1}$ bond leads to the relations

$$
\begin{aligned}
S_{x z}\left(\mathbf{D}_{1, \mathrm{LU}}\right) & =\mathbf{D}_{1, \mathrm{LO}}, \\
S_{x z}\left(\mathbf{D}_{1, \mathrm{LO}}\right) & =\mathbf{D}_{1, \mathrm{LU}}, \\
S_{x z}\left(\mathbf{D}_{1, \mathrm{RU}}\right) & =\mathbf{D}_{1, \mathrm{RO}}, \\
S_{x z}\left(\mathbf{D}_{1, \mathrm{RO}}\right) & =\mathbf{D}_{1, \mathrm{RU}}, \\
R S_{y}\left(\mathbf{D}_{1, \mathrm{LU}}\right) & =\mathbf{D}_{1, \mathrm{RO}}, \\
R S_{y}\left(\mathbf{D}_{1, \mathrm{LO}}\right) & =\mathbf{D}_{1, \mathrm{RU}}, \\
R S_{y}\left(\mathbf{D}_{1, \mathrm{RU}}\right) & =\mathbf{D}_{1, \mathrm{LO}}, \\
R S_{y}\left(\mathbf{D}_{1, \mathrm{RO}}\right) & =\mathbf{D}_{1, \mathrm{LU}} .
\end{aligned}
$$

To clarify the properties of $\mathbf{D}_{1}$ we start with an arbitrary vector

$$
\mathbf{D}_{1, \mathrm{LU}}=c_{x} \mathbf{e}_{x}+c_{y} \mathbf{e}_{y}+c_{z} \mathbf{e}_{z},
$$

where $\mathbf{e}_{\mu}$ are unit vectors in the directions indicated by the subscript and $c_{\mu}$ are real coefficients. Applying Eqs. (A2a) and (A2e) to this ansatz for $\mathbf{D}_{1, \mathrm{LU}}$ we obtain

$$
\begin{aligned}
& \mathbf{D}_{1, \mathrm{LO}}=c_{x} \mathbf{e}_{x}-c_{y} \mathbf{e}_{y}+c_{z} \mathbf{e}_{z}, \\
& \mathbf{D}_{1, \mathrm{RO}}=-c_{x} \mathbf{e}_{x}+c_{y} \mathbf{e}_{y}-c_{z} \mathbf{e}_{z} .
\end{aligned}
$$

The first condition determines that the $x$ and $z$ components are uniform while the $y$ component alternates along the ladder. The second condition indicates that all three components have odd parity since the translation to $\mathbf{D}_{1, \mathrm{RU}}$ changes the sign of the $y$ component as well so that all coefficients acquire a negative sign.

In the same way, we investigate $\mathbf{D}_{2}$. Applying both symmetry operations to $\mathbf{D}_{2}$ yields

$$
\begin{aligned}
S_{x z}\left(\mathbf{D}_{2, \mathrm{LU}}\right) & =\mathbf{D}_{2, \mathrm{LU}}, \\
S_{x z}\left(\mathbf{D}_{2, \mathrm{LO}}\right) & =\mathbf{D}_{2, \mathrm{LO}}, \\
S_{x z}\left(\mathbf{D}_{2, \mathrm{RU}}\right) & =\mathbf{D}_{2, \mathrm{RU}}, \\
S_{x z}\left(\mathbf{D}_{2, \mathrm{RO}}\right) & =\mathbf{D}_{2, \mathrm{RO}}, \\
R S_{y}\left(\mathbf{D}_{2, \mathrm{LU}}\right) & =\mathbf{D}_{2, \mathrm{RO}}, \\
R S_{y}\left(\mathbf{D}_{2, \mathrm{LO}}\right) & =\mathbf{D}_{2, \mathrm{RU}}, \\
R S_{y}\left(\mathbf{D}_{2, \mathrm{RU}}\right) & =\mathbf{D}_{2, \mathrm{LO}}, \\
R S_{y}\left(\mathbf{D}_{2, \mathrm{RO}}\right) & =\mathbf{D}_{2, \mathrm{LU}} .
\end{aligned}
$$

Again, we start from the general ansatz

$$
\mathbf{D}_{2, \mathrm{LU}}=d_{x} \mathbf{e}_{x}+d_{y} \mathbf{e}_{y}+d_{z} \mathbf{e}_{z} \text {. }
$$

Using Eq. (A5a) we easily see that the $y$ component has to vanish. In contrast, using Eq. (A5e) does not lead to an unambiguous solution because we obtain

$$
\mathbf{D}_{2, \mathrm{RO}}=-d_{x} \mathbf{e}_{x}-d_{z} \mathbf{e}_{z} \text {. }
$$

Each component can fulfill this condition in two different ways. Either the component alternates along the ladder with even parity or it is uniform along the ladder with odd parity. Thus, the $\mathbf{D}_{2}$ vector is generally expressed by the superposition of both possibilities

$$
\begin{aligned}
& D_{2}^{x}=D_{2, a}^{x}+D_{2, u}^{x}, \\
& D_{2}^{z}=D_{2, a}^{z}+D_{2, u}^{z},
\end{aligned}
$$

where subscript $a$ stands for "alternating" and $u$ for "uniform."
TABLE I. Behavior of the sign of the $D$ components along the legs of the spin ladder and their parity with respect to the symmetry $S_{x y}$ [reflection with respect to the center line of the spin ladder; see Fig. 1(c) of the main text]. $D$ components which are not listed are forbidden due to the selection rules of Moriya [37].

\begin{tabular}{lcc}
\hline \hline Couplings & Along the legs & Parity \\
\hline$D_{0}^{y}$ & alternating & odd \\
$D_{1}^{x}$ & uniform & odd \\
$D_{1}^{y}$ & alternating & odd \\
$D_{1}^{z}$ & uniform & odd \\
$D_{2, a}^{x}$ & alternating & even \\
$D_{2, u}^{x}$ & uniform & odd \\
$D_{2, a}^{z}$ & alternating & even \\
$D_{2, u}^{z}$ & uniform & odd \\
$D_{3}^{y}$ & alternating & N/A \\
\hline \hline
\end{tabular}

Considering the fact that the differences between the copper ions are small [35] we may neglect them altogether which allows us to conclude [24] $D_{2}^{x}=D_{2, u}^{x}$ and $D_{2}^{z}=D_{2, a}^{z}$. Thus, we conclude that the uniform $x$ component and the alternating $z$ component predominate. Arbitrary components as in Eq. (A8) are allowed, but decisive contributions only come from the alternating even-parity $z$ component and the uniform odd-parity $x$ component.

Note that we neglect potential differences between $\mathbf{D}_{2}$ on the $J_{2}$ bond and $\mathbf{D}_{2}^{\prime}$ on the $J_{2}^{\prime}$ bond because they have odd parity and do not contribute on the bilinear level anyway. The potential differences in the ensuing symmetric $\Gamma$ terms are neglected as well because of their barely measurable impact.

The results of the symmetry analysis are collected in Table I. Since the $\Gamma$ couplings result from the $D$ couplings according to Eq. (3) in the main article one can establish a similar table for the $\Gamma$ components based on Table I. The property of being alternating-odd corresponds to a minus sign while uniform-even corresponds to a plus sign in the DM components. Thus by multiplying \pm 1 to the DM components in Eq. (3) one arrives at the resulting properties of the $\Gamma$ components.

Finally, we remark that the orientation of the $\mathbf{D}_{3}$ vector, which belongs to the interladder coupling, is analogous to the $\mathbf{D}_{0}$ vector. The $\mathbf{D}_{3}$ vector couples two adjacent ladders contributing to the transversal dispersion. No parity can be defined because the reflection about the center line refers to a symmetry within each ladder separately.

\section{APPENDIX B: CONTINUOUS UNITARY TRANSFORMATIONS AND THE MATRIX REPRESENTATION OF THE BILINEAR HAMILTON OPERATOR}

The starting point of the present results is a continuous unitary transformation (CUT) applied to solve the isotropic spin ladder. The aim of the CUT is to systematically derive an effective tractable Hamilton operator $\mathcal{H}^{\text {eff }}$ from a complicated initial Hamilton operator $\mathcal{H}^{\text {init }}$. An approach which has proven to be very useful is to derive $\mathcal{H}^{\text {eff }}$ in second 
quantization in terms of creation and annihilation operators of the elementary excitations seen as quasiparticles. In the case of disordered quantum antiferromagnets these quasiparticles are often triplons [40]. Terms which change the number of quasiparticles are rotated away $[62,63]$, or at least some of them depending on the quantity one is interested in; for details see Ref. [64]. In this way, the ground state becomes the vacuum of quasiparticles and their dispersion can be taken from the hopping of quasiparticles, and so on.

In principle, a unitary transformation is a change of basis and can be done in one step. But is has turned out that it is more appropriate to perform the basis change continuously because this warrants renormalizing properties of the transformation. The processes between states with large energy differences are eliminated first; see for instance the Introduction of Ref. [42]. Thus, we consider a flow parameter $l \in[0, \infty]$, which takes the Hamiltonian continuously from $\mathcal{H}^{\text {init }}$ to $\mathcal{H}^{\text {eff }}$ by

$$
\mathcal{H}(l)=U(l) \mathcal{H}^{\text {init }} U^{\dagger}(l)
$$

with the initial condition $U(l=0)=\mathbb{1}$. The flow equation of the Hamiltonian is given by the differential equation

$$
\partial_{l} \mathcal{H}(l)=[\eta(l), \mathcal{H}(l)]
$$

with the anti-Hermitian infinitesimal generator $\eta(l)=$ $\left[\partial_{l} U(l)\right] U(l)$. Equation (B2) sets up a system of coupled differential flow equations for the prefactors of the operators appearing in $\mathcal{H}(l)$. To reduce the number of differential equations for tractability without significant loss of information an appropriate truncation scheme is required. There are various truncation schemes in use. For our calculations we use the directly evaluated enhanced perturbative CUT (deepCUT) [42]. Its truncation scheme is determined by a formally small expansion parameter $x$ and a target quantity, i.e., the quantity one intends to compute in a certain order $n$ of $x$. All terms in the flow equations required to compute the target quantity in order $n$ are kept; all others are omitted. Then the resulting set of flow equations is solved without further approximation such that the final result

$$
\mathcal{H}^{\text {eff }}=U(\infty) \mathcal{H}^{\text {init }} U^{\dagger}(\infty)
$$

contains contributions up to infinite order, but only those up to order $n$ are exact.
Since a CUT is a change of basis, observables $O$ must be transformed to the new basis as well. To do so a set of differential equations from

$$
\partial_{l} O=[\eta(l), O(l)]
$$

is derived with the same infinitesimal generator $\eta$ as the one used for transforming the Hamiltonian. In the limit $l \rightarrow \infty$ one obtains the effective observable $O^{\text {eff }}$ as well. We transform spin operators, so that we can express any additional coupling which is not part of the isotropic spin ladders, for instance the Dzyaloshinksii-Moriya terms or the interladder coupling, in terms of triplons.

In our case, the target quantity is the isotropic dispersion of the triplons and the $1: n$ generator is used for the CUT. The 1 : $n$ generator decouples the subspaces containing zero and one triplon from all other subspaces. It does not aim to decouple the sectors with two or more triplons. For determining the triplon dispersions this is sufficient [64].

The general expression in Nambu representation of the complete bilinear Hamiltonian in momentum space is given up to unimportant constants by

$$
\begin{aligned}
\mathcal{H} & =\frac{1}{2} \sum_{k, l} \mathbf{a}_{k, l}^{\dagger} \mathcal{M}_{k, l} \mathbf{a}_{k, l} \quad \text { with } \\
\mathcal{M}_{k, l} & =\left[\begin{array}{cc}
A(k, l) & B(k, l) \\
B^{\dagger}(k, l) & A^{\top}(-k,-l)
\end{array}\right]=\mathcal{M}_{k, l}^{\dagger}
\end{aligned}
$$

and the 12-dimensional Nambu spinor $\mathbf{a}_{k, l}=$ $\left(\mathbf{t}_{k, l}^{\top}, \mathbf{t}_{k+\pi, l}^{\top}, \mathbf{t}_{-k,-l}^{\dagger}, \mathbf{t}_{-k-\pi,-l}^{\dagger}\right)^{\top}$, see Eq. (5) in the main text, using $\mathbf{t}_{k, l}=\left(t_{k, l}^{x}, t_{k, l}^{y}, t_{k, l}^{z}\right)^{\top}$ and $\mathbf{t}_{k, l}^{\dagger}=\left(t_{k, l}^{x, \dagger}, t_{k, l}^{y, \dagger}, t_{k, l}^{z, \dagger}\right)$. Note that the sum in (B5a) runs over all values of $l \in[0,2 \pi)$ (lattice constant set to unity) in the Brillouin zone while it runs only over the values $k \in[0, \pi)$, i.e., over half the Brillouin zone. The reason is that the above Nambu spinor addresses $k$ and $k+\pi$ simultaneously.

The $12 \times 12$ matrix $\mathcal{M}_{k, l}$ is composed of the two $6 \times 6$ matrices $A$ and $B$ which are again made up by $3 \times 3$ matrices:

$$
A(k, l)=\left(\begin{array}{cc}
A_{1}(k)+B_{1}(k, l) & B_{2}(k, l) \\
B_{2}^{\dagger}(k, l) & A_{1}(k+\pi)+B_{1}(k+\pi, l)
\end{array}\right),
$$

$B(k, l)=\left(\begin{array}{cc}B_{1}(k, l) & B_{2}(k, l) \\ B_{2}^{\dagger}(k, l) & B_{1}(k+\pi, l)\end{array}\right)$.

The $3 \times 3$ matrices are derived to be

$$
\begin{aligned}
A_{1}(k) & =\left(\begin{array}{ccc}
\omega_{0}(k) & i h_{z} & -i h_{y} \\
-i h_{z} & \omega_{0}(k) & i h_{x} \\
i h_{y} & -i h_{x} & \omega_{0}(k)
\end{array}\right), \\
B_{1}(k, l) & =\left(\begin{array}{ccc}
F^{x}(k, l) & 0 & \Gamma_{1}^{x z}(k)+\Gamma_{2}^{x z}(k) \\
0 & F^{y}(k, l) & 0 \\
\Gamma_{1}^{x z}(k)+\Gamma_{2}^{x z}(k) & 0 & F^{z}(k, l)
\end{array}\right), \\
B_{2}(k, l) & =\left(\begin{array}{cccc}
0 & -i\left(\Gamma_{1}^{x y}(k)-D_{2}^{z}(k)\right) & -i D_{3}^{y}(k, l) \\
-i\left(\Gamma_{1}^{x y}(k)+D_{2}^{z}(k)\right) & 0 & -i\left(\Gamma_{1}^{y z}(k)-D_{2}^{x}(k)\right) \\
i D_{3}^{y}(k, l) & -i\left(\Gamma_{1}^{y z}(k)+D_{2}^{x}(k)\right) & 0
\end{array}\right) .
\end{aligned}
$$


TABLE II. The coefficients $\omega_{\delta}$ in order to describe the dispersion of the isotropic spin ladder as well as the prefactors $a_{\delta}$ to transform the spin operators are calculated by using the deepCUT method for the ratios $J_{1} / J_{0}=1.2$ and $J_{2} / J_{1}=0.9$. The values for higher $\delta$ are small enough to be neglected.

\begin{tabular}{lcc}
\hline \hline$\delta$ & $\omega_{\delta}$ & $a_{\delta}$ \\
\hline 0 & 1.5499384208488 & 0.3874491109155713 \\
1 & 0.358817770492231 & -0.05165001704799924 \\
2 & 0.524739087510573 & -0.08095884805094124 \\
3 & -0.209722209664048 & 0.03713614889687351 \\
4 & -0.160344853773972 & 0.0219291397751164 \\
5 & 0.0967516245738429 & -0.01719462494862808 \\
6 & 0.010462389004026 & -0.004727305201296136 \\
7 & -0.0347043572019398 & 0.01024208259455439 \\
8 & 0.000112462598212057 & -0.001628782296091526 \\
9 & 0.0139297388647789 & -0.00497492501969249 \\
10 & -0.00637707478352971 & 0.002315960919757644 \\
11 & -0.00403742286524941 & 0.001621270078823474 \\
12 & 0.00429559542625067 & -0.001835116321222724 \\
13 & 0.000461321168694168 & \\
\hline \hline
\end{tabular}

The dispersion of the isotropic spin ladder is calculated by the deepCUT method [24,42] yielding

$$
\omega_{0}(k)=\sum_{\delta=0}^{13} \omega_{\delta} \cos (\delta k) .
$$

The coefficients $\omega_{\delta}$ are given in Table II. Similarly, the transformation of the spin operators to triplon operators,

$$
\begin{aligned}
S_{0, L}^{\mu} & =-S_{0, R}^{\mu} \\
& =\sum_{\delta=-12}^{12} a_{|\delta|}\left(t_{\delta}^{\mu}+t_{\delta}^{\mu, \dagger}\right)+\text { bilinear and higher terms }
\end{aligned}
$$

yields the amplitudes $a_{\delta}$ also given in Table II. The spin operators are labeled with subscript left $(L)$ and right $(R)$ spin in a dimer referring to the two legs of each ladder. Bilinear or higher products of triplon operators are neglected in our approach to the transformation of the spin operator. The Fourier transform

$$
a(k):=\sum_{\delta=-12}^{12} a_{|\delta|} \cos (\delta k)
$$

yields the momentum-dependent amplitude $a(k)$ which appears generically in effective triplon Hamiltonians $[24,65,66]$. The Hamiltonian also includes a general uniform magnetic field $\mathbf{h}=\left(h_{x}, h_{y}, h_{z}\right)^{\top}$ given by $\mathcal{H}_{\text {Zeeman }}=-\mathbf{h} \sum_{i} \mathbf{S}_{i}$.

Further variables introduced for clarity are

$$
\begin{aligned}
F^{\mu}(k, l)= & d(k, l)+\Gamma_{0}^{\mu \mu}(k)+\Gamma_{1}^{\mu \mu}(k)+\Gamma_{2}^{\mu \mu}(k) \\
& +\Gamma_{3}^{\mu \mu}(k, l) \text { with } \quad \mu \in\{x, y, z\}
\end{aligned}
$$

and

$$
\begin{aligned}
d(k, l) & =-2 J_{3} \cos (l) a^{2}(k), \\
\Gamma_{0}^{\mu \mu}(k) & =-2 \Gamma_{0}^{\mu \mu} a^{2}(k),
\end{aligned}
$$

$$
\begin{aligned}
\Gamma_{1}^{\mu \mu}(k) & =4 \Gamma_{1}^{\mu \mu} a^{2}(k) \cos (k), \\
\Gamma_{2}^{\mu \mu}(k) & =4 \Gamma_{2}^{\mu \mu} a^{2}(k) \cos (2 k), \\
\Gamma_{3}^{\mu \mu}(k, l) & =-2 \Gamma_{3}^{\mu \mu} a^{2}(k) \cos (l), \\
\Gamma_{1}^{x y}(k) & =4 \Gamma_{1}^{x y} a(k) a(k+\pi) \sin (k), \\
\Gamma_{1}^{y z}(k) & =4 \Gamma_{1}^{y z} a(k) a(k+\pi) \sin (k), \\
\Gamma_{1}^{x z}(k) & =4 \Gamma_{1}^{x z} a(k)^{2} \cos (k), \\
\Gamma_{2}^{x z}(k) & =4 \Gamma_{2}^{x z} a(k)^{2} \cos (2 k), \\
D_{2, a}^{z}(k) & =4 D_{2, a}^{z} a(k) a(k+\pi) \sin (2 k), \\
D_{2, a}^{x}(k) & =4 D_{2, a}^{x} a(k) a(k+\pi) \sin (2 k), \\
D_{3}^{y}(k, l) & =-2 D_{3}^{y} a(k) a(k+\pi) \sin (l) .
\end{aligned}
$$

We point out that the notation employed here for the wave vector components $k, l$ differs from the one in Ref. [24] where reciprocal lattice units were used. The difference is a factor $\pi$ or $2 \pi$, respectively, as the lattice constant is set to unity.

Inspecting the above matrices one realizes that for zero magnetic field the slightly simpler form

$$
\mathcal{M}_{k, l}=\left[\begin{array}{ll}
A(k, l) & B(k, l) \\
B(k, l) & A(k, l)
\end{array}\right]
$$

holds. The eigenenergies and eigenmodes are obtained by a bosonic Bogoliubov transformation [46] from the $t$ operators to the normal $b$ operators. This requires the diagonalization of the matrix

$$
\widetilde{\mathcal{M}}_{k, l}:=\eta \mathcal{M}_{k, l}
$$

where the metric $\eta$ is the diagonal matrix

$$
\eta=\operatorname{diag}\left(1_{1}, \ldots, 1_{6},-1_{7}, \ldots,-1_{12}\right) .
$$

The resulting Hamiltonian reads $\mathcal{H}=$ $\sum_{n, k, l} \omega_{n}(k, l) b_{n, k, l}^{\dagger} b_{n, k, l}$, where the index $n$ labels the six different modes at given wave vector $(k, l)$. The normal bosonic operators read

$$
\begin{aligned}
b_{n, k, l}^{\dagger}= & \sum_{\mu=x, y, z}\left(u_{n, k, l}^{\mu} t_{k, l}^{\mu, \dagger}+\tilde{u}_{n, k, l}^{\mu} t_{k+\pi, l}^{\mu, \dagger}\right. \\
& \left.-v_{n, k, l}^{\mu} t_{-k,-l}^{\mu}-\tilde{v}_{n, k, l}^{\mu} t_{-k-\pi,-l}^{\mu}\right),
\end{aligned}
$$

where $u$ and $v$ with and without the tilde are generally complex prefactors. The annihilation operator follows by Hermitian conjugation.

\section{APPENDIX C: SYMPLECTIC PRODUCT AND BERRY PHASE FOR BOSONS}

The Berry phase in quantum mechanics is defined by the complex phase of the scalar product between two quantum states [67]. Thus, the key ingredient is an appropriate scalar product. In order to be independent of a particular basis for quantum states we aim at lifting the definition of topological phases from the level of quantum states to operators in second quantization. In Appendix B, we obtained the normal bosonic creation operator (B16). With the prefactors occurring in this equation we define a generalized ket by these prefactors,

$$
|\mathbf{k}, n\rangle\rangle:=\left(\mathbf{u}_{n, k, l}^{\top}, \tilde{\mathbf{u}}_{n, k, l}^{\top}, \mathbf{v}_{n, k, l}^{\top}, \tilde{\mathbf{v}}_{n, k, l}^{\top}\right)^{\top},
$$


which is a column vector with 12 components. The boldface symbols such as $\mathbf{u}$ stand for three-dimensional column vectors with components $u^{x}, u^{y}$, and $u^{z}$. Then, we define the symplectic product by

$$
\begin{aligned}
\left\langle\left\langle\mathbf{k}_{1}, n_{1} \mid \mathbf{k}_{2}, n_{2}\right\rangle\right\rangle:= & \left(\mathbf{u}_{n_{1}, k_{1}, l_{1}}^{\dagger}, \tilde{\mathbf{u}}_{n_{1}, k_{1}, l_{1}}^{\dagger}, \mathbf{v}_{n_{1}, k_{1}, l_{1}}^{\dagger}, \tilde{\mathbf{v}}_{n_{2}, k_{2}, l_{2}}^{\dagger}\right) \eta \\
& \times\left(\mathbf{u}_{n_{2}, k_{2}, l_{2}}^{\top}, \tilde{\mathbf{u}}_{n_{2}, k_{2}, l_{2}}^{\top}, \mathbf{v}_{n_{2}, k_{2}, l_{2}}^{\top}, \tilde{\mathbf{v}}_{n_{2}, k_{2}, l_{2}}^{\top}\right)^{\top} \\
= & \mathbf{u}_{n_{1}, k_{1}, l_{1}}^{\dagger} \mathbf{u}_{n_{2}, k_{2}, l_{2}}+\tilde{\mathbf{u}}_{n_{1}, k_{1}, l_{1}}^{\dagger} \tilde{\mathbf{u}}_{n_{2}, k_{2}, l_{2}} \\
& -\mathbf{v}_{n_{1}, k_{1}, l_{1}}^{\dagger} \mathbf{v}_{n_{2}, k_{2}, l_{2}}-\tilde{\mathbf{v}}_{n_{1}, k_{1}, l_{1}}^{\dagger} \tilde{\mathbf{v}}_{n_{2}, k_{2}, l_{2}} .
\end{aligned}
$$

We highlight the so far unnoted fact that $\widetilde{\mathcal{M}}_{k, l}$, in contrast to $\mathcal{M}_{k, l}$, see Eq. (B14), is self-adjoint with respect to this symplectic product implying the well-known facts that its eigenvalues are real and that creation and annihilation operators of different eigenvalues commute.

The above formalism is formulated on the level of second quantization. Here we want to elucidate its formal properties further. To be as general as possible, we consider a set of bosonic annihilation operators $a_{j}$ and creation operators $a_{j}^{\dagger}$ with $j \in\{1,2, \ldots, m\}$. A general linear combination $c$ reads

$$
c:=\sum_{j=1}^{m}\left(u_{j} a_{j}^{\dagger}-v_{j} a_{j}\right),
$$

where $c$ is not normalized and it is not specified whether it is a creation or annihilation operator. Then, we define the corresponding generalized ket by the column vector

$$
|c\rangle\rangle:=\left(u_{1}, \ldots, u_{m}, v_{q}, \ldots, v_{m}\right)^{\top}=\underline{c} .
$$

Sometimes the vector notation $\underline{c}$ is more convenient than the ket notation. Axiomatically, we can define the symplectic product between two kets $|c\rangle\rangle$ and $\left.\left|c^{\prime}\right\rangle\right\rangle$ by

$$
\begin{aligned}
\left\langle\left\langle c \mid c^{\prime}\right\rangle\right\rangle & :=\sum_{j=1}^{m}\left(u_{j}^{*} u_{j}^{\prime}-v_{j}^{*} v_{j}^{\prime}\right) \\
& =\underline{c}^{\dagger} \eta \underline{c}^{\prime},
\end{aligned}
$$

where the diagonal $2 m \times 2 m$ matrix $\eta=\operatorname{diag}\left(1_{1}, \ldots, 1_{m}\right.$, $\left.-1_{m+1}, \ldots,-1_{2 m}\right)$ is used as a metric with $\eta^{2}=\mathbb{1}$. This sort of "generalized scalar product" runs under several names in the literature such as "quasiscalar product" or "parascalar product", and the conserving transformation as "quasiunitary" or "paraunitary" $[17,29,68-70]$. We prefer to avoid the term "scalar product" completely because it suggests semipositivity, but use "symplectic" instead which is the established attribute for a metric with equal number of positive and negative directions; see for instance the symplectic structure of phase space in analytical mechanics.

It is easy to verify that a conventional Hermitian matrix $M=M^{\dagger}$ is not self-adjoint with respect to Eqs. (C5). But $\eta M$ is self-adjoint due to

$$
\begin{aligned}
\left\langle\left\langle c \mid \eta M c^{\prime}\right\rangle\right\rangle & =\underline{c}^{\dagger} \eta \eta M \underline{c}^{\prime} \\
& =\underline{c}^{\dagger} M \underline{c}^{\prime}, \\
\left\langle\left\langle\eta M c \mid c^{\prime}\right\rangle\right\rangle & =\underline{c}^{\dagger} M \eta \eta \underline{c}^{\prime} \\
& =\underline{c}^{\dagger} M \underline{c}^{\prime} .
\end{aligned}
$$

Alternatively, one can also start from

$$
\left\langle\left\langle c \mid c^{\prime}\right\rangle\right\rangle:=\left[c^{\dagger}, c^{\prime}\right]
$$

which obviously yields an expression identical to Eqs. (C5). The inequality $\langle\langle c \mid c\rangle\rangle>0$ tells us that $c$ is an unnormalized creation operator while $\langle\langle c \mid c\rangle\rangle<0$ tells us that it is an unnormalized annihilation operator.

The following question is imminent at this stage: Can one relate Eqs. (C5) and Eq. (C7) to the conventional scalar product between quantum states? The answer is ambiguous: it depends. If there is a general ground state, i.e., a vacuum $|0\rangle$ annihilated by all annihilation operators $b$ considered (here the linear combinations $b$ have to be annihilation operators), then the following relation between the standard scalar product $\left\langle 0\left|b^{\prime} b^{\dagger}\right| 0\right\rangle$ in Fock space for two one-particle states and the above-defined symplectic product holds:

$$
\begin{aligned}
\left\langle 0\left|b^{\prime} b^{\dagger}\right| 0\right\rangle & =\left\langle 0\left|\left(b^{\prime} b^{\dagger}-b^{\dagger} b^{\prime}\right)\right| 0\right\rangle \\
& =\left[b^{\prime}, b^{\dagger}\right],
\end{aligned}
$$

where the last line is precisely definition (C7) equivalent to (C5). Indeed, this situation is a very common one in multiband systems where $|0\rangle$ is the vacuum with respect to all bosons at all values of $\mathbf{k}$. Then one retrieves the Berry connection (10) and the Berry phase (11) for paths through the Brillouin zone in the main text.

But we stress that the identity (C8) does not hold if an external control parameter $\lambda$ is varied which changes the vacuum as well. Then the Berry phase for a path from $\lambda=0$ to $\lambda=\lambda_{1}$ reads

$$
\begin{aligned}
\Omega= & \mathbf{i} \int_{0}^{\lambda_{1}}\left\langle 0(\lambda)\left|b(\lambda) \partial_{\lambda} b^{\dagger}(\lambda)\right| 0(\lambda)\right\rangle d \lambda \\
= & \mathbf{i} \int_{0}^{\lambda_{1}}\left[\left\langle 0(\lambda)\left|b(\lambda)\left\{\partial_{\lambda} b^{\dagger}(\lambda)\right\}\right| 0(\lambda)\right\rangle\right. \\
& \left.+\langle 0(\lambda)| b(\lambda) b^{\dagger}(\lambda)\left\{\partial_{\lambda}|0(\lambda)\rangle\right\}\right] d \lambda \\
= & \mathbf{i} \int_{0}^{\lambda_{1}}\left[\left\langle 0(\lambda)\left|\left[b(\lambda),\left\{\partial_{\lambda} b^{\dagger}(\lambda)\right\}\right]\right| 0(\lambda)\right\rangle\right. \\
& \left.+\langle 0(\lambda)|\left\{\partial_{\lambda}|0(\lambda)\rangle\right\}\right] d \lambda \\
= & \Omega_{\mathrm{exc}}\left(\lambda_{1}\right)+\Omega_{\mathrm{vac}}\left(\lambda_{1}\right),
\end{aligned}
$$

where two contributions are identified:

$$
\begin{aligned}
& \Omega_{\mathrm{exc}}\left(\lambda_{1}\right):=\mathbf{i} \int_{0}^{\lambda_{1}}\left\langle 0(\lambda)\left|\left[b(\lambda),\left\{\partial_{\lambda} b^{\dagger}(\lambda)\right\}\right]\right| 0(\lambda)\right\rangle d \lambda \\
& \Omega_{\mathrm{vac}}\left(\lambda_{1}\right):=\mathbf{i} \int_{0}^{\lambda_{1}}\langle 0(\lambda)|\left\{\partial_{\lambda}|0(\lambda)\rangle\right\} d \lambda
\end{aligned}
$$

One contribution, $\Omega_{\mathrm{exc}}$, results from the bosonic excitation and equals what one obtains using the symplectic product. The other contribution, $\Omega_{\mathrm{vac}}$, is the Berry phase of the vacuum. For paths in the Brillouin zone the analogous result has been derived in Ref. [71] where, however, the vacuum contribution should not occur because the global vacuum of the system does not depend on momentum.

The bottom line is that for topological properties defined in the Brillouin zone the symplectic product yields a Berry phase 
identical to the conventional definition. In more general cases, however, the variation of the vacuum matters as well.

We corroborate this conclusion by repeating Berry's original adiabatic approach in the bosonic Fock space. Let us assume that the bilinear Hamiltonian depends on the parameter $\lambda$, which may parametrize a path in the Brillouin zone or may be an external control parameter. It is varied slowly from 0 to 1, i.e., $\lambda=t / T$ for $t \in[0, T]$ with $T \rightarrow \infty$. The Hamiltonian is generally given by the matrix $\mathcal{M}(\lambda)$ [46]; for an example see Eq. (4) in the main article. At each value of $\lambda$ the ket $|n(\lambda)\rangle$ parametrizes the creation of a boson in the $n$th eigenmode. Hence the equation

$$
\eta \mathcal{M}(\lambda)|n(\lambda)\rangle=\omega_{n}(\lambda)|n(\lambda)\rangle
$$

is fulfilled. We assume the eigenmodes to be non-degenerate for clarity. The adiabatic ansatz, see for instance Ref. [72], for the solution $\left|\psi_{n}(t)\right\rangle$ close to the instantaneous eigenstate $\left|\phi_{n}(\lambda)\right\rangle:=b_{n}^{\dagger}(\lambda)|0(\lambda)\rangle$ reads

$$
\left|\psi_{n}(t)\right\rangle=\exp [-\mathbf{i} \Theta(t)]\left[\left|\phi_{n}(\lambda(t))\right\rangle+(1 / T)|\perp\rangle\right],
$$

where the correction $(1 / T)|\perp\rangle$ is small in $1 / T$ and perpendicular to $\left|\phi_{n}(\lambda(t))\right\rangle$. Inserting this ansatz in the Schrödinger equation $\mathbf{i} \partial_{t}\left|\psi_{n}(t)\right\rangle=H\left|\psi_{n}(t)\right\rangle$ yields

$$
\begin{aligned}
H\left|\psi_{n}(t)\right\rangle= & \left(\partial_{t} \Theta\right)\left|\psi_{n}(t)\right\rangle+\exp [-\mathbf{i} \Theta(t)] \frac{\mathbf{i}}{T} \partial_{\lambda}\left|\phi_{n}(\lambda)\right\rangle \\
& + \text { perpendicular terms. }
\end{aligned}
$$

Next, we multiply with $\left\langle\phi_{n}(\lambda)\right|$ from the left to obtain

$$
\omega_{n}(\lambda)+E_{0}(\lambda)=\partial_{t} \Theta+\frac{1}{T} \partial_{\lambda}\left(\Omega_{\mathrm{exc}}(\lambda)+\Omega_{\mathrm{vac}}(\lambda)\right),
$$

where $E_{0}$ is the ground state energy and we used the result of the calculation (C9). Integrating from $\Theta(t=0)=0$ to $t=T$ yields

$$
\Theta(T)=T \int_{0}^{1}\left[\omega_{n}(\lambda)+E_{0}(\lambda)\right] d \lambda-\Omega_{\mathrm{exc}}(1)-\Omega_{\mathrm{vac}}(1) .
$$

This is the usual result for Berry phases in an adiabatic setting. The first term on the right-hand side of Eq. (C14) represents the dynamic phase and the second term $\Omega_{\mathrm{exc}}(1)+\Omega_{\mathrm{vac}}(1)$ is the Berry phase. Clearly, there is a contribution from the excitation and potentially from the ground state, i.e., the bosonic vacuum. Again, if the ground state is a global vacuum applying to all bosons it does not change as a function of $\lambda$. Then there is no vacuum Berry phase, i.e., $\Omega_{\mathrm{vac}}=0$. This is the case for topological phases determined in the Brillouin zone.

\section{APPENDIX D: NUMERICAL CALCULATION OF THE ZAK PHASE}

Only in rare cases, the analytical determination of the Zak phase is possible. In particular for higher-dimensional problems, for instance the 12-dimensional extended model considered for $\mathrm{BiCu}_{2} \mathrm{PO}_{6}$, a numerical approach is needed. The first step is to discretize the contour of integration. As an example for determining the phase from $k=0$ to $k=2 \pi$ we use $k_{i}=\frac{2 \pi i}{N}$ with $i=0,1, \ldots, N-1$ (lattice constant is set to unity). It is straightforward to determine the eigenmodes $\left.\left|n, k_{i}\right\rangle\right\rangle$ numerically. But the numerical choice of phase at each momentum $k_{i}$ is arbitrary so that we cannot rely on a continuous evolution and hence an approximation of

$$
\Omega_{n}=\mathbf{i} \int_{0}^{2 \pi}\left\langle\left\langle n, k\left|\partial_{k}\right| n, k\right\rangle\right\rangle d k
$$

does not work. A well-established solution $[73,74]$ consists in using the Wilson loop

$$
\Omega_{n}=-\operatorname{Im} \sum_{i=0}^{N-1} \ln \left(\left\langle\left\langle n, k_{i} \mid n, k_{i+1}\right\rangle\right\rangle\right) \bmod 2 \pi
$$

instead, where $\left.\left.\left|n, k_{0}\right\rangle\right\rangle=\left|n, k_{N}\right\rangle\right\rangle$ holds because the loop is closed. We stress that in the above formula the gauge, i.e., the choice of the phase, of each eigenmode does not matter because it cancels out. Regauging each eigenmode arbitrarily

$$
\left.\left.\left|n, k_{j}\right\rangle\right\rangle \rightarrow \widetilde{\left.\left|n, k_{j}\right\rangle\right\rangle}=\exp \left(\mathbf{i} \varphi_{j}\right)\left|n, k_{j}\right\rangle\right\rangle
$$

does not alter the outcome of Eq. (D2) because each eigenmode appears once as ket and once as bra.

An alternative variant of the above approach relies on the idea of parallel transport. The eigenmode $\left.\left|n, k_{j}\right\rangle\right\rangle$ serves as a reference state for $\left.\left|n, k_{j+1}\right\rangle\right\rangle$. If their symplectic product reads

$$
\left\langle\left\langle n, k_{j} \mid n, k_{j+1}\right\rangle\right\rangle=z=|z| \exp \left(-\mathbf{i} \varphi_{j+1}\right)
$$

we regauge $\left.\left|n, k_{j+1}\right\rangle\right\rangle$ such that it becomes as parallel as possible to $\left.\left|n, k_{j}\right\rangle\right\rangle$. Obviously, this is achieved by

$$
\left.\left.\left.\left|n, k_{j+1}\right\rangle\right\rangle \rightarrow\left|\widetilde{n, k_{j+1}}\right\rangle\right\rangle=\exp \left(\mathbf{i} \varphi_{j+1}\right)\left|n, k_{j+1}\right\rangle\right\rangle .
$$

This procedure is iterated recursively from $j=0$ to $j=$ $N-2$. The next and final step for $j=N-1$ yields $\varphi_{N}$, but the corresponding regauging (D5) is not possible because the phase of $\left.\left.\left|n, k_{0}\right\rangle\right\rangle=\left|n, k_{N}\right\rangle\right\rangle$ is fixed already. Then the total sum (D2) simply reduces to

$$
\Omega_{n}=-\operatorname{Im} \ln \left(\left\langle\left\langle n, k_{N-1} \mid n, k_{0}\right\rangle\right\rangle\right),
$$

since all regauged products are real and positive so that the Zak phase corresponds to

$$
\Omega_{n}=\varphi_{N} .
$$

The attractive feature of this second variant is that it reveals the geometric character of the Berry phases. They stem from the parallel transport in the U(1) principal fiber bundle of the manifold given by the eigenmodes as a function of momenta.

\section{APPENDIX E: BERRY CURVATURE IN $\mathrm{BiCu}_{2} \mathrm{PO}_{6}$}

The Zak phase is computed from an integral of the Berry connection $\mathcal{A}_{n \text {,sym }}(\mathbf{k})$ in Eq. (7) in the main text which depends on the gauge used for the normal bosonic operators. Changing the gauge changes the Berry connection in its dependence on the wave vector $\mathbf{k}$ without changing the integral, i.e., the Zak phase. Hence, it is a priori not clear from which regions in the Brillouin zone the topological behavior results. Two arguments, however, show that the topological non-trivial Zak phase results from the vicinity of the minima of the triplon dispersions.

First, we consider the process of switching on the DM interactions. Without them there is no finite Zak phase. The DM terms couple the $x$ mode at $k$ with the $y$ mode at $k+\pi$ and 
(a)

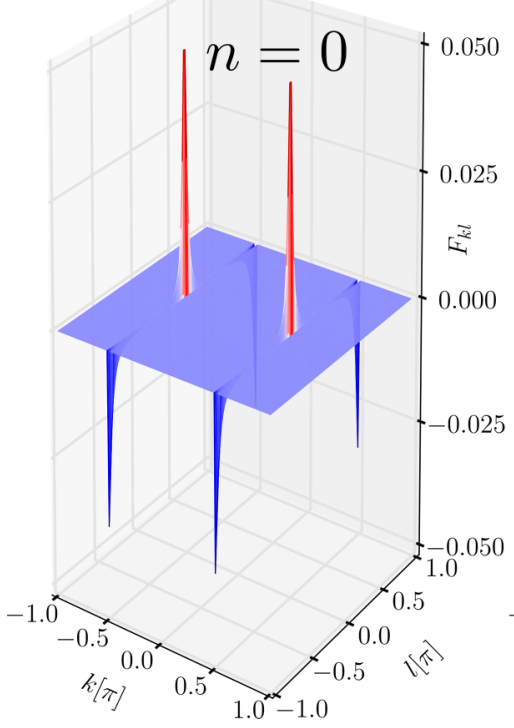

(b)

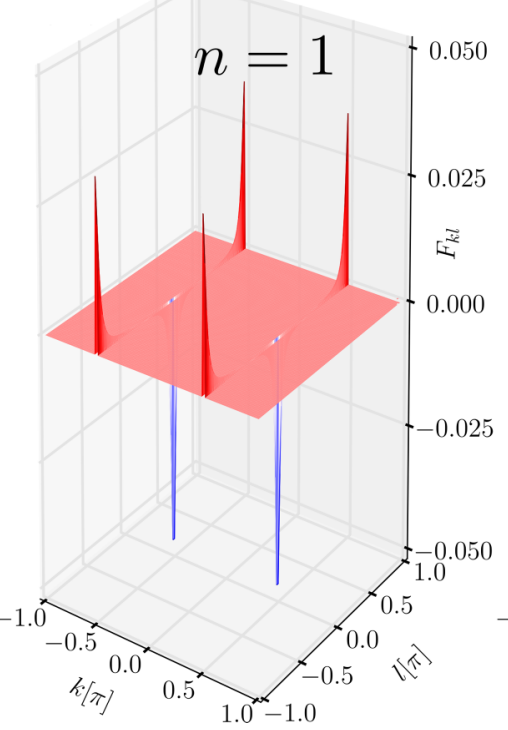

(c)

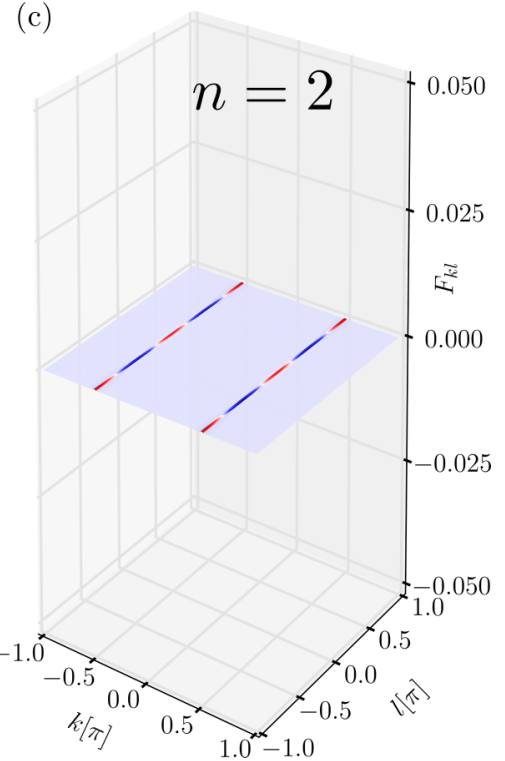

FIG. 9. The Berry curvature of the three lowest triplon dispersions calculated for $J_{0}=9.4 \mathrm{meV}, J_{3}=1.5 \mathrm{meV}, J_{1}=1.2 J_{0}, J_{2}=1.09 J_{0}$, $D_{1}^{x}=0.58 J_{0}, D_{1}^{y}=0.73 J_{0}, D_{2, a}^{z}=-0.02 J_{0}, D_{3}^{y}=0.02 J_{0}, h_{x}=0.1 \mathrm{~T}$, and $h_{y}=0.1 \mathrm{~T}$. The curvature in panels (a) and (b) shows high peaks which are located in the vicinity of $k= \pm \pi / 2$. In contrast, panel (c) shows only a negligible curvature which corresponds to the topologically trivial $z$ mode.

vice versa. The degeneracy between the two modes of the $x-y_{\pi}$ modes at $k=\pi / 2$ and at $k=3 \pi / 2$ is lifted by the DM terms (see green lines in Fig. 2 of the main text). The same holds for the two modes of the $y-x_{\pi}$ modes (see blue lines in Fig. 2 of the main text). The significant band mixing and splitting at $k=$ $\pi / 2$ and at $k=3 \pi / 2$ is the driving mechanism of the finite Zak phase. Hence, the vicinity of these points in the Brillouin zone is decisive for the topologically non-trivial behavior.

Second, we study the Berry curvature $F$

$$
\begin{aligned}
F_{n}(k, l)= & \mathbf{i}\left[\left(\partial_{k}\langle\langle k, l, n|)\left(\partial_{l}|k, l, n\rangle\right\rangle\right)\right. \\
& \left.-\left(\partial_{l}\langle\langle k, l, n|)\left(\partial_{k}|k, l, n\rangle\right\rangle\right)\right],
\end{aligned}
$$

which is a gauge-independent quantity and thus suitable to investigate from which region non-trivial behavior results. All modes in the calculated dispersion of $\mathrm{BiCu}_{2} \mathrm{PO}_{6}$ show degenerate crossing points at $k=\pi / 2$ and $k=3 \pi / 2$. These degeneracies prevent the unambiguous definition of the Berry curvature. In order to be able to define an unambiguous curvature we add a small magnetic field in the $x$ and $y$ direction $\vec{h}=h(1,1,0)^{\top}$, which lifts the degeneracies leading to six well-separated bands. Mathematically, the required magnetic field can be chosen infinitesimally small; for computational stability we chose $h=0.1 \mathrm{~T}$. The Zak phases do not change upon adding this small magnetic field. The Berry curvature of the three lowest bands $n=\{0,1,2\}$ is shown in Fig. 9. While the summation over the whole Brillouin zone vanishes so that the Chern number is zero, it is evident that the strong topological twists result from the vicinity of the original degeneracy points around $k=\pi / 2$ and $k=3 \pi / 2$. This is the region where the triplon dispersions are low and the agreement with experiment is very good. This piece of evidence supports the validity of the minimal model for the determination of topological phases in spite of missing features such as the downturns in the dispersions close to two-triplon continua [23].

\section{APPENDIX F: WINDING NUMBER $w$}

In the main text, we focused on the Zak phase because it is based on a Berry phase and can be defined for any one-dimensional system regardless of symmetries. But there is another often considered topological invariant in one-dimensional systems, namely the winding number [32,51,52,75].

The winding number counts the number of windings around a point in a two-dimensional plane of parameters. For this concept to make sense, the Hamiltonian must have an additional symmetry, conventionally called chiral symmetry, so that in the eigenbasis of the chiral symmetry the Hamiltonian matrix becomes block off-diagonal. A pseudospin representation can be used to illustrate the winding tracking the end point of the pseudospin vector upon varying the wave vector from $k=0$ to $k=2 \pi$. The chiral symmetry pins the vector to a two-dimensional plane of parameters; see Refs. [32,51,52].

Such a chiral symmetry operator can be found for the minimal model of $\mathrm{BiCu}_{2} \mathrm{PO}_{6}$, i.e., ignoring the difference between the copper sites. But we were not able to find a chiral symmetry operator for the extended model accounting for different copper sites. The winding numbers $w$ found for the $x-y_{\pi}$ and $x_{\pi}-y$ mode both take the non-trivial value $w=-1$ upon increasing $k$ from 0 to $2 \pi$. We emphasize, however, that the Zak phase is a far more general concept because its definition and computation do not require an additional symmetry.

In the case of the established minimal model for $\mathrm{BiCu}_{2} \mathrm{PO}_{6}$ the Hamiltonian shows the above-discussed additional chiral symmetry allowing us to calculate the winding number if minor additional approximations are made. Here we discuss the details of this calculation.

In the minimal model with $D_{3}^{y}=0$, the $12 \times 12$ matrix in Eq. (6) in the main article or in Eq. (B5a) in Appendix B 
can be split into $4 \times 4$ matrices simplifying the subsequent analysis which is performed similarly to the one in Ref. [52]. To this end, we focus on the $x$ mode and its coupling to the $y_{\pi}$ mode. Since all couplings which are proportional to the $4 \times 4$ identity matrix do not alter the eigenmodes they do not alter the topological properties and are therefore neglected. The coupling contributions proportional to $\sigma_{x} \otimes \mathbb{1}$ only lead to small variations of the energy dispersion and we neglect them in a simplifying approximation. We checked that their omission has no impact on the Zak phase. We expect that the winding number similarly is not changed by the couplings proportional to $\sigma_{x} \otimes 1$, because their effect on the dispersion is small compared to the gap between the $x-y_{\pi}$ modes (see green lines in Fig. 2). Thus the topological invariants remain conserved because no gap has closed. The same is assumed for the inclusion of small $D_{3}^{y}$. Thus, for simplicity, we consider the Hamiltonian of a single ladder

$$
\mathcal{H}=\frac{1}{2} \sum_{k} \mathbf{a}_{k}^{\dagger} \mathcal{M}_{k} \mathbf{a}_{k}
$$

with the Nambu spinor $\mathbf{a}_{k}=\left(t_{k}^{x}, t_{k+\pi}^{y}, t_{-k}^{x, \dagger}, t_{-k-\pi}^{y, \dagger}\right)^{\top}$ and the $4 \times 4$ matrix

$$
\mathcal{M}_{k}=\left[\begin{array}{ll}
C(k) & C(k) \\
C(k) & C(k)
\end{array}\right],
$$

where the $2 \times 2$ matrix $C$ is parametrized by Pauli matrices $\sigma=\left(\sigma_{x}, \sigma_{y}, \sigma_{z}\right)$ :

$$
\begin{aligned}
C(k) & =\mathbf{d}(k) \cdot \sigma, \\
d_{x}(k) & =0, \quad d_{y}(k)=\Gamma_{1}^{x y}(k)-D_{2}^{z}(k), \\
d_{z}(k) & =\frac{1}{2} \sum_{i=0}^{2}\left[\Gamma_{i}^{x x}(k)-\Gamma_{i}^{y y}(k+\pi)\right] .
\end{aligned}
$$

Then, the chiral symmetry operator is easy to identify as $\mathbb{1} \otimes \sigma_{x}$. It fulfils the anticommutator $\left\{\mathbb{1} \otimes \sigma_{x}, \mathcal{M}_{k}\right\}=0$.
In order to calculate the winding number we transform the Hamiltonian into the eigenbasis of the chiral symmetry operator. This is achieved by the unitary transformation

$$
U=\frac{1}{\sqrt{2}}\left(\begin{array}{rrrr}
1 & 0 & 1 & 0 \\
1 & 0 & -1 & 0 \\
0 & 1 & 0 & 1 \\
0 & 1 & 0 & -1
\end{array}\right)
$$

In this basis, the Hamiltonian matrix $\eta \mathcal{M}_{k}$ with the metric $\eta=\sigma_{z} \otimes \mathbb{1}$ has a block off-diagonal form

$$
\begin{aligned}
\widetilde{\mathcal{M}}_{k} & =U^{\dagger}\left(\eta \mathcal{M}_{k}\right) U \\
& =\left[\begin{array}{cc}
0 & D_{1}(k) \\
D_{1}^{*}(k) & 0
\end{array}\right] .
\end{aligned}
$$

The matrix $D_{1}(k)$ is given by

$$
D_{1}(k)=\left(\begin{array}{cc}
d_{3}(k)+\mathbf{i} d_{2}(k) & d_{3}(k)+\mathbf{i} d_{2}(k) \\
-d_{3}(k)-\mathbf{i} d_{2}(k) & -d_{3}(k)-\mathbf{i} d_{2}(k)
\end{array}\right)
$$

and the winding number [76] is calculated by

$$
w=\frac{1}{8 \pi \mathbf{i}} \oint_{\mathrm{BZ}} d k \operatorname{Tr}_{2}\left[D^{-1} \partial_{k} D-\left(D^{\dagger}\right)^{-1} \partial_{k} D^{\dagger}\right]
$$

with $D=\left[D_{1}(k)+D_{1}^{\top}(k)\right] / 2$. By construction, the winding number is quantized to integer values $w \in \mathbb{Z}$. For the investigated mode we find $w=-1$.

The same analysis can be performed for the $y$ mode coupled to the $x_{\pi}$ mode yielding the same winding number. In contrast, the $z$ mode only displays the trivial winding number $w=0$ because it does not couple with another mode. Hence, it cannot be twisted or wound in any way.

A chiral symmetry of the general $12 \times 12$ matrix including all possible contributions could not be identified so that we could not define a winding number in general.
[1] F. D. M. Haldane, Rev. Mod. Phys. 89, 040502 (2017).

[2] M. König, S. Wiedmann, C. Brüne, A. Roth, H. Buhmann, L. W. Molenkamp, X.-L. Qi, and S.-C. Zhang, Science 318, 766 (2007).

[3] M. Z. Hasan and C. L. Kane, Rev. Mod. Phys. 82, 3045 (2010).

[4] X.-L. Qi and S.-C. Zhang, Rev. Mod. Phys. 83, 1057 (2011).

[5] A. B. Bernevig and T. L. Hughes, Topological Insulators and Topological Superconductors (Princeton University Press, Princeton, 2013).

[6] Y. Ando, J. Phys. Soc. Jpn. 82, 102001 (2013).

[7] C.-Z. Chang, J. Zhang, X. Feng, J. Shen, Z. Zhang, M. Guo, K. Li, Y. Ou, P. Wei, L.-L. Wang et al., Science 340, 167 (2013).

[8] N. Goldman, J. C. Budich, and P. Zoller, Nat. Phys. 12, 639 (2016).

[9] M. C. Rechtsman, J. M. Zeuner, Y. Plotnik, Y. Lumer, D. Podolsky, F. Dreisow, S. Nolte, M. Segev, and A. Szameit, Nature (London) 496, 196 (2013).

[10] T. Jacqmin, I. Carusotto, I. Sagnes, M. Abbarchi, D. D. Solnyshkov, G. Malpuech, E. Galopin, A. Lemaître, J. Bloch, and A. Amo, Phys. Rev. Lett. 112, 116402 (2014).
[11] Z. Yang, F. Gao, X. Shi, X. Lin, Z. Gao, Y. Chong, and B. Zhang, Phys. Rev. Lett. 114, 114301 (2015).

[12] C. Kane and T. Lubensky, Nat. Phys. 10, 39 (2014).

[13] R. Süsstrunk and S. D. Huber, Science 349, 47 (2015).

[14] H. Katsura, N. Nagaosa, and P. A. Lee, Phys. Rev. Lett. 104, 066403 (2010).

[15] Y. Onose, T. Ideue, H. Katsura, Y. Shiomi, N. Nagaosa, and Y. Tokura, Science 329, 297 (2010).

[16] R. Matsumoto and S. Murakami, Phys. Rev. Lett. 106, 197202 (2011).

[17] R. Shindou, R. Matsumoto, S. Murakami, and J.-I. Ohe, Phys. Rev. B 87, 174427 (2013).

[18] L. Zhang, J. Ren, J.-S. Wang, and B. Li, Phys. Rev. B 87, 144101 (2013).

[19] R. Chisnell, J. S. Helton, D. E. Freedman, D. K. Singh, R. I. Bewley, D. G. Nocera, and Y. S. Lee, Phys. Rev. Lett. 115, 147201 (2015).

[20] J. Romhányi, K. Penc, and R. Ganesh, Nat. Commun. 6, 6805 (2015).

[21] M. Malki and K. P. Schmidt, Phys. Rev. B 95, 195137 (2017). 
[22] P. A. McClarty, F. Krüger, T. Guidi, S. F. Parker, K. Refson, A. Parker, D. Prabhakaran, and R. Coldea, Nat. Phys. 13, 736 (2017).

[23] K. W. Plumb, K. Hwang, Y. Qiu, L. W. Harriger, G. E. Granroth, A. I. Kolesnikov, G. J. Shu, F. C. Chou, C. Ruegg, Y. B. Kim et al., Nat. Phys. 12, 224 (2016).

[24] L. Splinter, N. A. Drescher, H. Krull, and G. S. Uhrig, Phys. Rev. B 94, 155115 (2016).

[25] K. Hwang and Y. B. Kim, Phys. Rev. B 93, 235130 (2016).

[26] R. S. K. Mong and V. Shivamoggi, Phys. Rev. B 83, 125109 (2011).

[27] A. M. Essin and V. Gurarie, Phys. Rev. B 84, 125132 (2011)

[28] M. Wada, S. Murakami, F. Freimuth, and G. Bihlmayer, Phys. Rev. B 83, 121310(R) (2011).

[29] S. Murakami, J. Phys.: Conf. Ser. 302, 012019 (2011).

[30] B.-J. Yang, M. S. Bahramy, and N. Nagaosa, Nat. Commun. 4, 1524 (2013)

[31] J. Zak, Phys. Rev. Lett. 62, 2747 (1989).

[32] P. Delplace, D. Ullmo, and G. Montambaux, Phys. Rev. B 84, 195452 (2011).

[33] M. Atala, M. Aidelsburger, J. T. Barreiro, D. Abanin, T. Kitagawa, E. Demler, and I. Bloch, Nat. Phys. 9, 795 (2013).

[34] F. Cardano, A. D’Errico, A. Dauphin, M. Maffei, B. Piccirillo, C. de Lisio, G. De Filippis, V. Cataudella, E. Santamato, L. Marrucci et al., Nat. Commun. 8, 15516 (2017).

[35] A. A. Tsirlin, I. Rousochatzakis, D. Kasinathan, O. Janson, R. Nath, F. Weickert, C. Geibel, A. M. Läuchli, and H. Rosner, Phys. Rev. B 82, 144426 (2010).

[36] K. W. Plumb, Z. Yamani, M. Matsuda, G. J. Shu, B. Koteswararao, F. C. Chou, and Y.-J. Kim, Phys. Rev. B 88, 024402 (2013).

[37] T. Moriya, Phys. Rev. 120, 91 (1960).

[38] L. Shekhtman, O. Entin-Wohlman, and A. Aharony, Phys. Rev. Lett. 69, 836 (1992).

[39] L. Shekhtman, A. Aharony, and O. Entin-Wohlman, Phys. Rev. B 47, 174 (1993).

[40] K. P. Schmidt and G. S. Uhrig, Phys. Rev. Lett. 90, 227204 (2003).

[41] S. Sachdev and R. N. Bhatt, Phys. Rev. B 41, 9323 (1990).

[42] H. Krull, N. A. Drescher, and G. S. Uhrig, Phys. Rev. B 86, 125113 (2012).

[43] F. Wegner, Ann. Phys. 506, 77 (1994).

[44] S. D. Głazek and K. G. Wilson, Phys. Rev. D 48, 5863 (1993).

[45] S. D. Głazek and K. G. Wilson, Phys. Rev. D 49, 4214 (1994).

[46] J.-P. Blaizot and G. Ripka, Quantum Theory of Finite Systems (MIT Press, Cambridge, 1986).

[47] H. Zhang, C.-X. Liu, X.-L. Qi, X. Dai, Z. Fang, and S.-C. Zhang, Nat. Phys. 5, 438 (2009).

[48] H.-Z. Lu1 and S.-Q. Shen, Front. Phys. 12, 127201 (2017).
[49] F. Liu and K. Wakabayashi, Phys. Rev. Lett. 118, 076803 (2017).

[50] T. L. Hughes, E. Prodan, and B. A. Bernevig, Phys. Rev. B 83, 245132 (2011).

[51] L. Li, C. Yang, and S. Chen, Europhys. Lett. 112, 10004 (2015).

[52] D. G. Joshi and A. P. Schnyder, Phys. Rev. B 96, 220405(R) (2017).

[53] B. Kramer and A. MacKinnon, Rep. Prog. Phys. 56, 1469 (1993).

[54] M. Calixto and E. Romera, J. Stat. Mech.: Theor. Exp. (2015) P06029.

[55] M. Malki and G. S. Uhrig, Europhys. Lett. 127, 27001 (2019).

[56] M. I. Molina, A. E. Miroshnichenko, and Y. S. Kivshar, Phys. Rev. Lett. 108, 070401 (2012).

[57] G. Corrielli, G. Della Valle, A. Crespi, R. Osellame, and S. Longhi, Phys. Rev. Lett. 111, 220403 (2013).

[58] Y. Baum, T. Posske, I. C. Fulga, B. Trauzettel, and A. Stern, Phys. Rev. Lett. 114, 136801 (2015).

[59] R. Verresen, N. G. Jones, and F. Pollmann, Phys. Rev. Lett. 120, 057001 (2018).

[60] W. P. Su, J. R. Schrieffer, and A. J. Heeger, Phys. Rev. Lett. 42 1698 (1979).

[61] K. P. Schmidt and G. S. Uhrig, Mod. Phys. Lett. B 19, 1179 (2005)

[62] C. Knetter and G. S. Uhrig, Eur. Phys. J. B 13, 209 (2000).

[63] C. Knetter, K. P. Schmidt, and G. S. Uhrig, J. Phys. A: Math. Gen. 36, 7889 (2003).

[64] T. Fischer, S. Duffe, and G. S. Uhrig, New J. Phys. 12, 033048 (2010).

[65] G. S. Uhrig, K. P. Schmidt, and M. Grüninger, Phys. Rev. Lett. 93, 267003 (2004).

[66] G. S. Uhrig, K. P. Schmidt, and M. Grüninger, J. Phys. Soc. Jpn. 74, 86 (2005).

[67] M. V. Berry, Proc. R. Soc. London, Ser. A 392, 45 (1984).

[68] J. H. P. Colpa, Physica A 93, 327 (1978).

[69] Y. Kawaguchia and M. Ueda, Phys. Rep. 520, 253 (2012).

[70] H. Kondo, Y. Akagi, and H. Katsura, Phys. Rev. B 99, 041110(R) (2019).

[71] V. Peano, M. Houde, C. Brendel, F. Marquardt, and A. A. Clerk, Nat. Commun. 7, 10779 (2016).

[72] G. S. Uhrig, Z. Phys. B 82, 29 (1991).

[73] F. Grusdt, D. Abanin, and E. Demler, Phys. Rev. A 89, 043621 (2014).

[74] F. Wilczek and A. Zee, Phys. Rev. Lett. 52, 2111 (1984).

[75] A. P. Schnyder, S. Ryu, A. Furusaki, and A. W. W. Ludwig, Phys. Rev. B 78, 195125 (2008).

[76] D. G. Joshi, K. Coester, K. P. Schmidt, and M. Vojta, Phys. Rev. B 91, 094404 (2015). 